\title{
Dynamical Quantities in Net Systems
}

\author{
Hartmann J. Genrich ${ }^{1}$ \\ Bonn, Germany
}

\begin{abstract}
The paper presents some known material of higher-level Petri nets in analogy to the dynamics of physical systems. Its aim is to widen the view on the role of the incidence matrix in the analysis of net systems. Rather than focusing on constant state quantities (S-invariants) and cyclic actions (T-invariants), i.e. on solutions of the homogeneous equation systems based on the incidence matrix, it studies dynamical quantities of net systems in general.
\end{abstract}

Keywords: Dynamics; Petri nets

\section{Introduction}

Petri nets are employed to a considerable extent in the analysis of systems with a combined continuousdiscrete dynamics which in particular result from the computerised control of engineering processes. This inevitably raises the question how net systems with their discrete states and changes are related to the classical models in physics and engineering based on infinitesimal changes of states.

The sequel is an attempt to present some known material of higher-level Petri nets ${ }^{2}$ from a particular perspective, namely in analogy to the dynamics of physical systems. It is also an attempt to complement, on a macroscopic level, the foundation that Carl Adam Petri has given to net theory in accordance with the laws of physics (see [Pet62, Pet82], for example).

Our purpose in looking at physics is to get orientation, not to reach it - like using the stars for navigation. Most likely there will be more irreconcilable differences than similarities between net systems and physical systems. However, both similarities and differences are equally important for understanding the role of Petri nets in engineering systems analysis.

In Mechanics, Kinematics describes the movement of a body as a sequence of spatial-geometrical positions, while Dynamics describes the same movement in terms of physical quantities that the body, in the course

\footnotetext{
1 This work was done while the author was with GMD - National Research Center for Information Technology (now Fraunhofer Gesellschaft). Part of it was funded by Deutsche Forschungsgemeinschaft, Priority Programme Analysis and Synthesis of ContinuousDiscrete Engineering Systems (KONDISK), Ref. No. GE 933/1-2.

Correspondence and offprint requests to: Hartmann J. Genrich, Peter-Ohlenhardt-Str. 5, D-53127 Bonn, Germany.

Email: hartmann.genrich@gmd.de

2 Throughout this paper, the term Petri nets denotes the higher-level standard models of net theory like Predicate/Transion nets, Coloured Petri nets or Algebraic Petri nets which trivially include conventional transition nets. A standard model is one that has been derived by net theoretical abstractions and constructions from the basic model, the elementary net systems of conditions and events, rather than being defined only in terms of simulating annotated graphs of boxes, circles and connectors.
} 
of moving, receives from and delivers to its environment (other bodies, fields). Hence the notion of an exchangeable quantity is essential to dynamics. The fundamental quantities that by their exchange control the movements are energy, linear momentum and angular momentum. The nature of the exchange of those quantities is particularly simple because it obeys universal conservation laws.

Dynamics does not depend on any geometrical view; it describes a process - a continuous sequence of states - as the receiving and delivering of physical (dynamical) quantities not necessarily proper to mechanics. This kind of description is of fundamental importance for all physics. The firm rules and principles that determine the dynamical description of physical systems and processes comprise a General Dynamics [Fal66].

Net theory also does not depend on any geometrical view - even though the graphical appearance of Petri nets is so prominent that it is often taken for the matter itself. As opposed to physics, however, which associates with every state quantity $X$ both its values (that constitute the states) and its infinitesimal changes $d X$ that constitute the processes, net theory describes the states of an object (system) as sets of coexisting state elements (conditions) and the processes as partially ordered sets of change elements (events) and treats state elements and change elements on an equal formal footing.

From elementary net systems of conditions and events, higher-level net models are derived through abstraction - like Predicate Transition Nets [GeL81] and Coloured Petri Nets [Jen92]. As computer-executable models, hierarchical coloured Petri nets [CPN96] are as powerful as higher-level programming languages, and as standard models of net theory they are subject to formal net theoretical analysis.

One particular aspect of the higher-level net analysis will be presented in this paper: the analysing of a system in terms of identifying dynamic quantities and studying their properties and relationships. This is tentatively called the dynamics point of view of Petri nets, in contrast to a kinematics point of view which deals, for example, with firing sequences and occurrence graphs.

The main part of this paper consists of a piecemeal confrontation of selected elements of net theory with sections of a presentation of the Dynamics of (classical rather than quantum mechanical) physical systems [Fal66]. ${ }^{3}$ The running example is the Petri net model of a simple production scheme. An Appendix briefly introduces an experimental computer-algebra tool for Petri nets that supports the necessary symbolic calculations.

The reader is assumed to have a certain familiarity with Petri nets in general and higher-level nets (PrT nets, CP nets) in particular. This paper is not meant as an introduction to Petri nets, nor to modelling of engineering systems with Petri nets, nor to the dynamics of physical systems.

\section{States and Quantities}

\subsection{Dynamics}

A physical system is a totality of states. The processes in which the system takes part appear as transitions between states. Among the quantities of a system there are some which are called state quantities or dynamical variables in the sequel. They are related to the states in the following way:

Definition. A dynamical variable of a physical system is a measuring rule that assigns to each state exactly one value.

Examples are displacement, force, momentum, energy; non-examples are work, heat or duration since they belong to processes rather than states.

For identifying the states one selects a maximal number of independent variables; each may vary while all the others remain unchanged. Such a selection is called a coordinate system; the number of coordinates is the number of degrees of freedom of a system. If one knows, in addition to the coordinates fixing the states, the values of all other, dependent variables in every state - as a function of the coordinates - the system is completely known.

Let $\Sigma^{a}$ and $\Sigma^{b}$ be two systems that have, among others, the variables $x^{a}$ and $x^{b}$. If $\Sigma^{a}$ and $\Sigma^{b}$ can be coupled in such a way that

$$
x^{a}+x^{b}=\text { const. }
$$

\footnotetext{
3 This presentation, although in German and a bit out of date, was chosen because with its intention and structure it was a perfect guideline for this paper.
} 


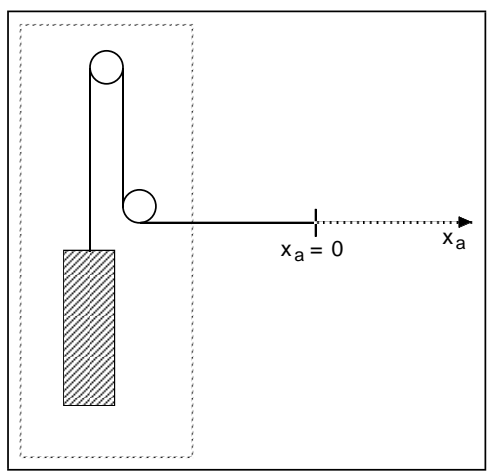

$\Sigma_{a}$ : Mass in the gravitational field

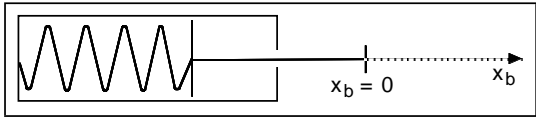

$\Sigma_{b}$ : Spring

Fig. 1. Two systems of the same type (displacement).

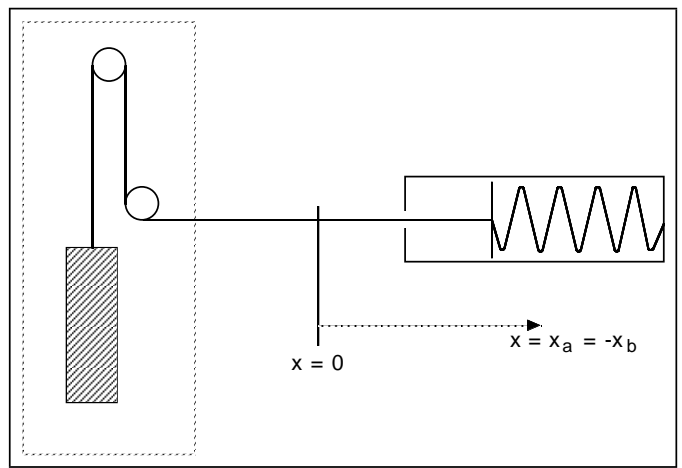

Fig. 2. Mass and spring coupled by exchange of coordinate $x$ (dimension length)

while $x^{a}$, or $x^{b}$, can vary freely, the variables $x^{a}$ and $x^{b}$ are called exchangeable. $\Sigma^{a}$ and $\Sigma^{b}$ allow an exchange of 'the' variable $x$; the amount of $x$ that one system loses is gained by the other.

Example. See Figs 1 and 2.

Definition. Two variables $x_{1}$ and $x_{2}$ are called comparable if they are exchangeable themselves or there is a third variable that is exchangeable with both.

Hence with (1), two variables are comparable iff they can be added, if they have the same dimension. Since (1) denotes an interaction between $\Sigma^{a}$ and $\Sigma^{b}$ we finally get:

Every physical variable represents a particular interaction between a system and its environment.

Let $\Sigma^{a}$ and $\Sigma^{b}$ be two systems with the same number of degrees of freedom. If their variables are in pairs comparable, $\Sigma^{a}$ and $\Sigma^{b}$ are said to be of the same type. The following principle is based upon experience. It is of fundamental importance to the dynamical description of physical systems and their processes.

Principle. If $\Sigma^{a}$ and $\Sigma^{b}$ are systems of the same type, any coordinate system $\left\{x_{1}, \ldots, x_{n}\right\}$ for $\Sigma^{a}$ is a coordinate system for $\Sigma^{b}$ as well. And conversely, if two systems have a coordinate system in common, they are of the same type.

In order to determine whether two given systems with $n$ degrees of freedom are of the same type, it is sufficient to find in each system $n$ independent variables such that they are pairwise comparable. 


\subsection{Net Theory}

A net system is a totality of states and changes. As opposed to physics, which describes a process as a continuous sequence of states (with time as parameter), net theory describes the states as sets of coexisting state elements (conditions) and the processes as sets of change elements (events) equipped with a partial order earlier. And while physics associates with each variable $x$ both its values (that constitute the states) and its infinitesimal changes $d x$ in a process, net theory treats states and changes on an equal formal footing.

Every state determines which events may happen next - are enabled - and which may have happened last. The set of events that may happen at a state $c$ is called the en-ability of $c$ and is denoted by $\mathscr{E}(c)$. Every event $e$ has a pre-set $e$ of conditions that cease to hold and a post-set $e^{\bullet}$ of conditions that begin to hold through an occurrence of $e$. This effect of an event also determines its en-ability. In its axiomatic foundation, general net theory [Pet77] states the following

Extensionality Principle. For every event $e$, both effect and possibility of occurring are uniquely determined by a pair of sets of conditions, $\left(\cdot e, e^{\bullet}\right)$. For any two states $c_{1}, c_{2}$, the following holds:

$$
c_{1} \stackrel{e}{\longrightarrow} c_{2} \Longleftrightarrow c_{1}-c_{2}=\cdot e \wedge c_{2}-c_{1}=e^{\bullet}
$$

Physical quantities are real-valued; more precisely, their values are given as the product of a physical unit (centimetre, gram, second,...) with a real number. In general, this is not the case for the quantities of net systems. Yet some minimal 'numbership' is required as well: it must be possible to add the values of a quantity to each other and to multiply them by scalars - usually integer numbers but rational or even real numbers are not excluded. A function that maps the states or the processes of a net system into some set of values is called a quantity only if it satisfies this requirement. Non-examples are all statements about states or processes; $3 \cdot$ true, for example, is not a truth value.

A structure whose elements can be added and multiplied by the elements of a ring, $A$, is a module, an abelian group, $M$, together with an operation of $A$ on $M$ such that for all $a, b \in A$ and $x, y \in M$ we have

$$
(a+b) x=a x+b x \quad a(x+y)=a x+a y
$$

The markings of a place $s$ of a higher-level Petri net, for example, belong to the module of all integer linear combinations of the elements of the data type (colour set) associated with $s$. Note that in the special case of conventional Petri nets, the module is the ring of integers itself.

Definition. A state quantity (process quantity) of a net system $\mathscr{N}$ is a function that maps the states (processes) of $\mathscr{N}$ into a module.

In the sequel, we shall encounter two kinds of modules as the value range of a quantity of a net system: (linear) combinations and weighted sets. Let $A$ be the ring of integer, rational or real numbers, and let $D$ be a set.

1. The module is the set of all formal linear combinations of elements of $D$ with coefficients from $A$. In this case, the elements of $D$, the generators of the module, will be called pieces.

2. The module is the set of all weighted sets over $D$ with weights in $A$, i.e. of all mappings of $D$ into $A$. For any weighted sets $u, v \in[D \rightarrow A]$, scalar $c \in A$ and element $d \in D$, we have

$$
\begin{aligned}
(u+v)(d) & =u(d)+v(d) \\
(c u)(d) & =c u(d)
\end{aligned}
$$

The ordering of $A$ induces a partial ordering of $[D \rightarrow A]$. For $u, v \in[D \rightarrow A]$ we have

$$
u \geqslant v \stackrel{\Delta}{\Longleftrightarrow} u(d) \geqslant v(d) \text { for all } d \in D
$$

Formally, combinations can be viewed as a special case of weighted sets, namely those which only for a finite number of elements have a non-zero weight. Conceptionally, however, and with respect to their usage we want to keep them separate.

If the values of two physical quantities $x_{1}$ and $x_{2}$ can be added, $x_{1}$ and $x_{2}$ are comparable; they have the same 'dimension'. For example, energy (a state quantity) and work (a process quantity) have the same 
dimension, mass length ${ }^{2} /$ time $^{2}$. In an analogous way, if $x$ is a quantity of a net system and $D$ is the set such that the values of $x$ are combinations or weighted sets over $D$, we call $D$ the dimension of $x$.

Example: quantity: commercial value of a commodity

dimension: money

pieces: the coins and bills of convertible currency

unit: owner's currency | a key currency

The marking of a place $s$ is an integer combination of pieces belonging to the colour set of $s$ (in this case, the pieces are called tokens traditionally). Hence, the places of a net system are state quantities, the values being the markings at the respective states. Since the markings can be varied independently and determine the states, the places are the coordinates of a net system. ${ }^{4}$ Two net systems whose places are in pairs comparable - have the same colour sets - are of the same type.

The places being the coordinates, net systems in general have quite a large number of coordinates in comparison to physical systems. However, the numerical solution of the differential equations describing the physical system usually also leads to equation systems with a very large number of variables. Net theory just makes a different use of those many variables that are a consequence of the discrete approach. ${ }^{5}$

\subsubsection{An Example}

Figure 3 together with Table 1 depict the running example of this paper: the Coloured Petri Net (CPN, [Jen92]) model of a simple production scheme using batch processing [GeH95]. Several products or substances pass through various stages of production according to their 'recipes'. The stages are identified with some components of the production site called devices. Hence a recipe is just a list of devices, for example $[D 1, D 2, D 3, D 1]$. Every incoming order designates a batch - the production of a certain integer quantity of the same substance. Depending on the availability of devices, several batches may be processed concurrently.

The declarations in Table 1 assign a formal meaning to the graphical presentation, Fig. 3, such that it can be executed by a tool like Design/CPN [CPN96]. Each place is associated with a data type, called its colour set; only tokens which belong its colour set may appear on the place. ${ }^{6}$ In Fig. 3 some colour sets are shown; they are the basic ones like Bch (batch), $D v c$ (device), $R c p$ (recipe) or $R d b$ (recipe database), for example. Others that are considered auxiliary are hidden; they can be derived from the adjacent arc annotations.

The system appears divided into three subsystems. The innermost one represents the production proper, the processing of a batch as a double iteration over recipe and multiplicity. It is surrounded by an administration that accepts the orders and delivers the results. Production and administration share the fused pairs of places, $\left(s_{1}, s_{1}^{\prime}\right),\left(s_{3}, s_{3}^{\prime}\right)$ and $\left(s_{4}, s_{4}^{\prime}\right)$. The shaded transitions, finally, are shared with an - invisible - environment that sends orders, excepts the deliveries, does the device maintenance and may develop new recipes. They are assumed to be shut off during simulation and analysis.

The annotations and global declarations of a Design/CPN model are derived from Standard ML [Pau91]. Brackets [,] denote lists, the constructor :: combines head and tail of a list, and the operator ${ }^{\wedge}$ concatenates $^{\wedge}$ two lists. The function rev reverses a list; set converts a list into a set. The CPN multi-set operators ' and \% denote the multiplication of a value by an integer scalar and by a Boolean coefficient, respectively. Transition guards and Boolean coefficients are lists of Boolean expressions.

The model is timed and uses parametric duration functions at the transitions. Tokens carry time stamps that indicate their earliest availability. The time stamps are set by the transitions relative to the simulation time according to their time regions and timed arc expressions (operator @ + ). In the sequel, time does not play an important role; see also Section 5.

To become familiar with the example, let us consider what happens with one of the tokens in the initial marking of place $s_{0}$. The work item (batch) $(X, 3)$, 'produce 3 units of substance $X$ ', appears on place $s_{0}$ as an incoming order. The processing of the batch begins with an occurrence of transition $t_{1}$ for the binding $\{c, r, x, n \leftarrow 3,[D 1, D 3], X, 0\}$. This takes the batch $(X, 3)$ from the incoming orders, $s_{0}$, reads the recipe for $X$, $r=[D 1, D 3]$, from the recipe database, $s_{7}$, and increases its counter $n$ of current usage, allocates the set of

\footnotetext{
4 They have an 'absolute zero'; the markings are non-negative.

5 Note that for this comparison the integer-valued places of the unfolded, equivalent conventional Petri net have to be considered.

6 The essence of such a colour set (in the mathematical sense) is that each element ('colour') has a finite representation, like the integers and unlike the reals, for example. In addition they are usually associated with some algebraic structure, and if they are compound the constituents of their elements can be determined by pattern matching.
} 


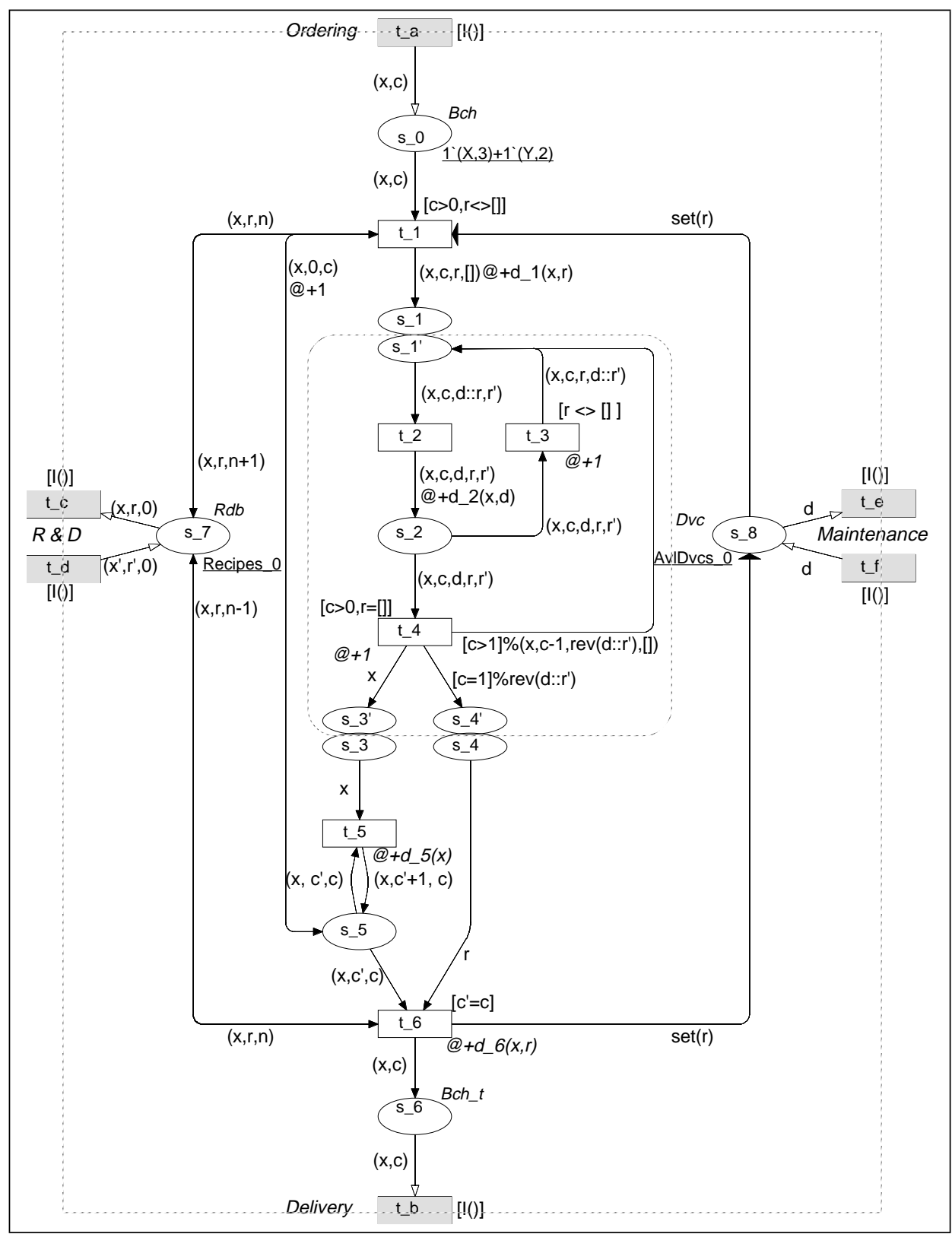

Fig. 3. A simple production scheme.

devices listed in $r$, set $(r)=1^{\circ} D 1+1^{\prime} D 3$, by removing it from the pool of available devices, $s_{8} . t_{1}$ also puts an empty container for $X$ 's for accumulating the finished units of a batch $s_{5}$ and initialises the status vector (substance, units still to produce, steps still to go, steps already done) on place $s_{1}$.

All this happens, at the chosen level of abstraction, as a single indivisible event - an occurrence of $t_{1}$. However, it may occur only if the binding for $c$ and $r$ satisfies the list of conditions stated in the guard of $t_{1},[c>0, r \neq[]]$, that is, if the count $c$ and the recipe $r$ are meaningful and non-trivial. And, of course, the corresponding tokens required must be present at the input places.

The identifiers $x, c, r, n$ are typed, individual variables called $C P N$ variables in Design/CPN. The CPN variables are declared in the global declarations (Table 1) that are part of the CPN model. A CPN variable occurs at a transition $t$ if it occurs freely in its guard expression or in one of the adjacent arc expressions. Its scope is $t$ or, more precisely, a single occurrence ('firing') of $t$ specified by a particular binding. In a single 
Table 1. Global declarations for the production scheme

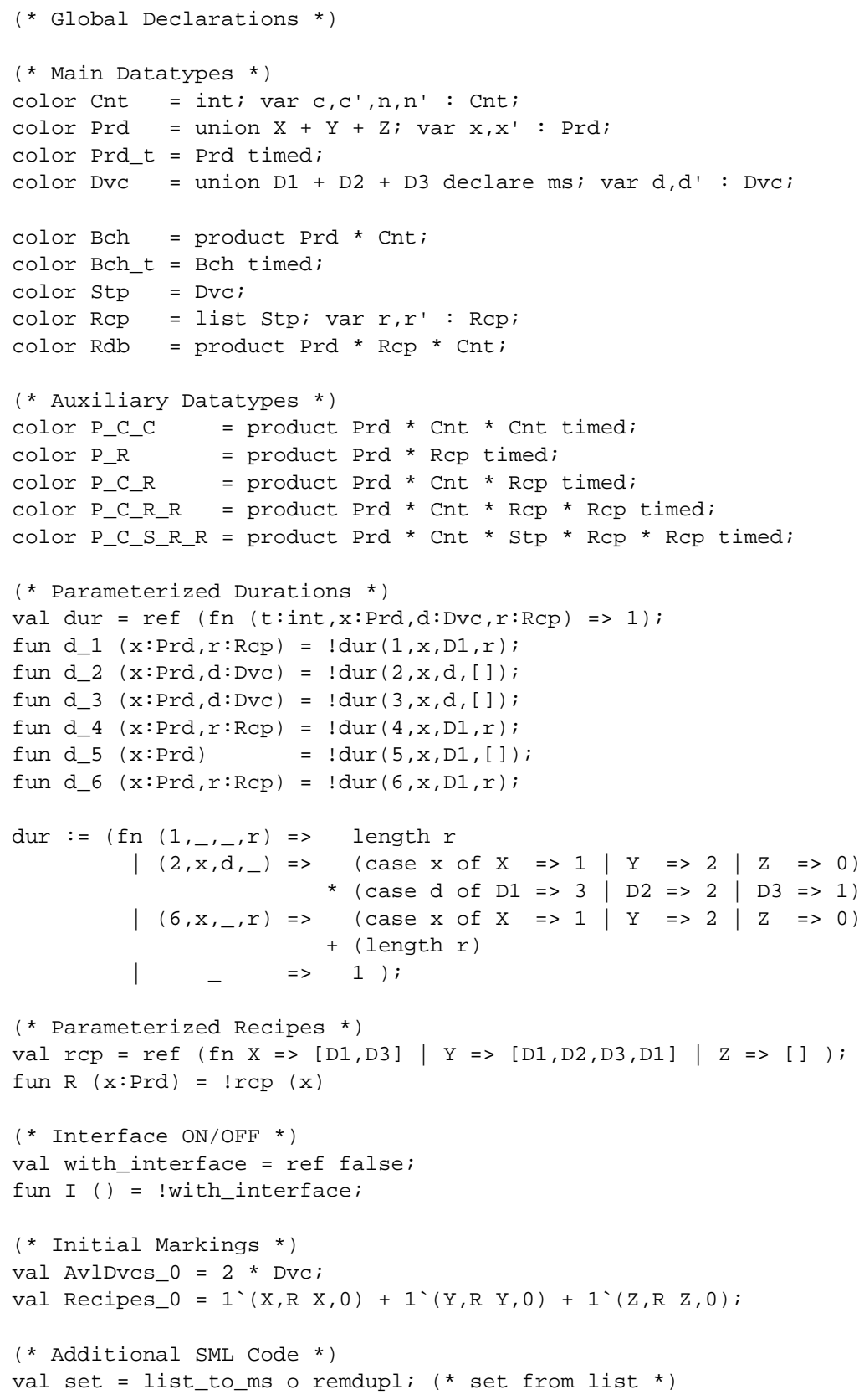

occurrence of $t$, all copies of the same individual variable at $t$ are assigned to the same value. The same variables may be bound, independently, in other transition occurrences, including other occurrences of the same transition (e.g. for the other batch, $(Y, 2)$, waiting on place $\left.s_{0}\right)$.

The identifiers $X, Y, Z$ and $D 1, D 2, D 3$, in contrast to individual variables, are constants of the net system. They are declared globally in the declarations to denote the different substances and devices. Also the identifiers Recipes ${ }^{0}$ and $A v l D v c s^{0}$ denoting the initial markings of the recipe database, $s_{7}$, and the pool of available devices, $s_{8}$, respectively, are syntactical constants, as well as all functions defined in ML like set, rev 
and the duration functions $d_{1}, \ldots, d_{5}$. Some of those 'constants', however, are more like parameters. Markings may be changed interactively between runs, and the definition of the durations contains a reference to a function dur that may be changed between runs as well.

Through transition $t_{1}$ one enters the kernel of the processing of a batch. It consists of two loops. The inner one is formed by transitions $t_{2}$ and $t_{3}$ and follows the recipe step by step until one unit of substance $X$ is done. A single production step is identified, for the sake of simplicity, utilising a particular device (resource). When one unit is done, $t_{4}$ puts it on place $s_{3}$ for accumulation at $s_{5}$. And, as long as more units have yet to be produced, $t_{4}$ also restores the recipe, decreases the count in the status vector, and enters another round; otherwise it keeps the recipe for control purposes on $s_{4}$.

Transition $t_{5}$ accumulates the single units of a substance in the corresponding container that was put on place $s_{5}$ by transition $t_{1}$. If the whole batch is done, $t_{6}$ puts the result ready for delivery on place $s_{6}$, decreases the count of recipe usages on place $s_{7}$ and returns the set of devices that were reserved for the batch in the beginning to place $s_{8}$.

The processing of the batch $(X, 3)$ alone takes 24 time units; at the end the token $(X, 3)$ on place $s_{6}$ carries the time stamp @ 24 if simulation starts at time zero. The processing of $(Y, 2)$ alone as well as the concurrent processing of both $(X, 3)$ and $(Y, 2)$ takes 55 time units; the available devices are sufficient to run both without interference.

\subsubsection{Combinations of Pieces}

The symbolic analysis of net systems deals with expressions for combinations of pieces, combinations of such expressions, and two kinds of transformations of expressions: substitutions and distributions. In essence it consists of calculating row sums and column sums of the transformed incidence matrix (see Section 2.2.3).

The arc expressions of a net system denote combinations of pieces. They map the set of bindings for the respective adjacent transition (transition 'colours') into the module of integer combinations of elements of the colour set of the respective adjacent place. Note that the so-called Boolean coefficients of arc expressions actually denote scalars. They map the truth value false onto zero (unit of addition) and true onto one (unit of multiplication).

$$
[\text { true }] \cdot x=x \quad[\text { false }] \cdot x=\mathbf{0} \quad\left[b_{1}, \ldots, b_{k}\right] \cdot x=\left[b_{1} \wedge \ldots \wedge b_{k}\right] \cdot x
$$

Let $D$ be a data type and let $u$ be an expression denoting a combination of pieces of $D$. We call $D$ the dimension of $u$. The scope of $u$ is assumed to be either a particular transition (e.g. if $u$ is an arc expressions) which determines the set of typed individual variables that can occur in $u$ or global if there are no free individual variables in $u$ (e.g. if $u$ denotes the initial marking of a place).

Expressions for combinations may be combined if they are comparable, i.e. if they have (1) the same dimension and (2) the same scope. The arc expressions around a single place - in one row of the incidence matrix - satisfy the first condition; their dimension is the colour set of that place. The arc expressions around a single transition - in one column of the incidence matrix - satisfy the second condition; their scope is that transition.

In order to form row sums and column sums the respective missing condition must be imposed by suitable transformations. There are two kinds of such transformations that will be used in the sequel:

1. Substitutions: that replace individual variables by individual terms within the given scope, i.e. observing the type constraints and not introducing new variables. The simultaneous substitution of variables $x_{i}$ by terms $t_{i}$ is written as $\left\{x_{1} \leftarrow t_{1}, \ldots, x_{n} \leftarrow t_{n}\right\}$, the symbol $\leftarrow$ indicating the binding and replacement.

2. Distributions: mappings that transform the pieces of the given data type $D$ into combinations of pieces of another data type $D^{\prime}{ }^{7}$ With possibly several argument patterns $p_{i}$ for pieces of $D$ to distinguish different cases, a distribution is written as $\left\{p_{1} \mapsto v_{1}|\ldots| p_{n} \mapsto v_{n}\right\}$, the symbol $\mapsto$ indicating the mapping.

Most often we write the application of a transformation to an expression as a product. ${ }^{8}$ Usually it is clear

\footnotetext{
7 'Distribution' because it usually decomposes a given piece and recombines (distributes) the constituents into a linear combination. 8 It was with respect to this product that K. Lautenbach and the author were mistaken when - after having discovered the PrT nets we tried to lift integer linear-algebraic techniques to the new level of abstraction. We considered the ring of polynomials over the set of arc expressions with integer coefficients as the proper generalisation of the ring of integers. We had some success - just enough to make us believe that we were on the right track [GeL81]. It was K. Jensen, however, who came up with the proper product - the product of functions [Jen81].
} 
from the context which factor is the expression and which is the transformation. If needed we use ordinary juxtaposition if the transformation is on the left and the operator $\odot$ if it is on the right.

$$
\tau u=\tau(u)=u \odot \tau
$$

Example. Let $c$ be of type integer, $x, y$ of type $A=\{\underline{a}, \underline{b}, \underline{c}\}$, and $r$ of type (A list).

$$
\begin{aligned}
\sigma & =\{c \leftarrow 3, r \leftarrow[a, b]\} & & \text { substitution } \\
\delta & =\{(c, r) \mapsto c \cdot r\} & & \text { distribution } \\
(c, r) \odot \sigma & =(3,[\underline{a}, \underline{b}]) & & \\
(c, r) \odot \delta & =c \cdot r & & \\
((c, r) \odot \sigma) \odot \delta & =((c, r) \odot \delta) \odot \sigma & & \sigma \text { and } \delta \text { commute } \\
& =3 \cdot[a, b] & &
\end{aligned}
$$

If distributions or substitutions are comparable, i.e. if the distributions have the same domain and range or the substitutions occur within the same scope, they can be combined:

$$
u \odot\left(\tau_{1}+\tau_{2}\right)=u \odot \tau_{1}+u \odot \tau_{2}
$$

A combination of distributions $\delta_{1}$ and $\delta_{2}$ is again a distribution. Since $\delta_{1}$ and $\delta_{2}$ are comparable, their argument patterns can be unified and then mapped onto the combined image. For example,

$$
\begin{aligned}
\delta_{1} & =\{(c, r) \mapsto c \cdot r\} \\
\delta_{2} & =\{(c,[x, y]) \mapsto c x \cdot y\} \\
\delta_{1}+\delta_{2} & =\{(c,[x, y]) \mapsto(c \cdot[x, y]+c x \cdot y)\}
\end{aligned}
$$

In order to combine the arc expressions around a place - to form a row sum - one has to get rid of all the individual variables first; they are local to the transitions. Similarly, in order to combine the arc expressions around a transition - to form a column sum - one has to transform them first such that they all get the same dimension.

Let $X=\left(X_{i}\right)_{i \in I}$ be a vector of expressions of the same dimension and let $B=\left(B_{i}\right)_{i \in I}$ be a vector of substitutions (transition bindings) such that each $B_{i}$ is within the scope of $X_{i}$ and eliminates its individual variables. Then the dot product of $X$ and $B$ is

$$
\langle X, B\rangle=\sum_{i \in I} X_{i} \odot B_{i}
$$

Let $X=\left(X_{i}\right)_{i \in I}$ be a vector of expressions of the same scope and let $V=\left(V_{i}\right)_{i \in I}$ be a vector of distributions having the same range and such that for each $i$ the domain of $V_{i}$ is the dimension of $X_{i}$. Then the dot product of $X$ and $V$ is

$$
\langle X, V\rangle=\sum_{i \in I} X_{i} \odot V_{i}
$$

To end this section on pieces, let us do a little arithmetic with pieces that themselves are numbers, e.g. integers. They are underlined to distinguish them from the scalar coefficients. According to our definitions, the combination $\underline{2}+\underline{3}$ is different from $\underline{5}$, and $\underline{2}+\underline{2}$ equals $2 \cdot \underline{2}$ rather than $\underline{4}$. However, if we apply the distribution $\zeta=\{x \mapsto x \cdot()\}$, then $(\underline{2}+\underline{3}) \odot \zeta=2 \cdot()+3 \cdot()=5 \cdot()$ and $(\underline{2}+\underline{2}) \odot \zeta=2 \cdot(2 \cdot())=4 \cdot()$.

\subsubsection{The Incidence Matrix}

In essence, this paper is concerned with representing a net system by its incidence matrix, usually denoted as $C$. The incidence matrix of our running example, the production scheme in Fig. 3, is shown in Table 2.

Notation. For a matrix $A=\left(A_{i}^{k}\right), A_{i}$ denotes the $i$ th row of $A$ and $A^{k}$ denotes the $k$ th column of $A$. $A^{\top}$ denotes the transpose of $A:\left(A^{\top}\right)_{k}^{i}=A_{i}^{k}$.

Dot product and matrix product are based upon the product between expressions and transformations (see (10) and (11)). Vectors are considered as one-column matrices.

$$
\langle U, V\rangle=\sum_{i} U_{i} \odot V_{i}=U^{\top} \cdot V
$$


Table 2. Incidence matrix of the production scheme (border transitions and time regions omitted)

\begin{tabular}{|c|c|c|c|c|c|c|c|}
\hline & $t_{1}$ & $t_{2}$ & $t_{3}$ & $t_{4}$ & $t_{5}$ & $t_{6}$ & $M^{0}$ \\
\hline $\mathscr{G}$ & $\begin{array}{l}{[c>0,} \\
r \neq[]]\end{array}$ & & {$[r \neq[]]$} & $\begin{array}{l}{[c>0,} \\
r=[]]\end{array}$ & {$[c \geqslant 0]$} & {$\left[c^{\prime}=c\right]$} & \\
\hline$s_{0}$ & $-(x, c)$ & & & & & & $\begin{array}{r}1^{\prime}(X, 3) \\
+1^{\prime}(Y, 2)\end{array}$ \\
\hline$s_{1}$ & $(x, c, r,[]])$ & $-\left(x, c, d:: r, r^{\prime}\right)$ & $\left(x, c, r, d:: r^{\prime}\right)$ & $\begin{array}{r}{[c>1] \%(x, c-1,} \\
\left.\operatorname{rev}\left(d:: r^{\prime}\right),[]\right)\end{array}$ & & & \\
\hline$s_{2}$ & & $\left(x, c, d, r, r^{\prime}\right)$ & $-\left(x, c, d, r, r^{\prime}\right)$ & $-\left(x, c, d, r, r^{\prime}\right)$ & & & \\
\hline$s_{3}$ & & & & $x$ & $-x$ & & \\
\hline$s_{4}$ & & & & {$[c=1] \% \operatorname{rev}\left(d:: r^{\prime}\right)$} & & $-r$ & \\
\hline$s_{5}$ & $(x, 0, c)$ & & & & $\begin{array}{c}-\left(x, c^{\prime}, c\right) \\
+\left(x, c^{\prime}+1, c\right)\end{array}$ & $-\left(x, c^{\prime}, c\right)$ & \\
\hline$s_{6}$ & & & & & & $(x, c)$ & \\
\hline$s_{7}$ & $\begin{array}{c}-(x, r, n) \\
+(x, r, n+1)\end{array}$ & & & & & $\begin{array}{c}-(x, r, n) \\
+(x, r, n-1)\end{array}$ & Recipes $^{0}$ \\
\hline$s_{8}$ & $-\operatorname{set}(r)$ & & & & & $\operatorname{set}(r)$ & $A v l D v c^{0}$ \\
\hline
\end{tabular}

Each row $C_{s}$ of the incidence matrix corresponds to a place $s$ and each column $C^{t}$ corresponds to a transition $t$. An entry $C_{s}^{t}$ is an expression denoting a combination of pieces, namely the change at place $s$ effected by an event at transition $t$. The minus sign distinguishes the input places of $t,{ }^{\bullet} t=$ pre $(t)$, from its output places, $t^{\bullet}=\operatorname{post}(t)$. Accordingly $C$ can be split into two matrices, ${ }^{\circ} C$ and $C^{\bullet}$, such that $C=-{ }^{\circ} C+C^{\bullet}$.

In addition there may be

- one row $\mathscr{G}$ with the guards of the transitions;

- one row $\mathscr{T}$ with the time increments of the transitions (if the model is timed);

- one column $\mathscr{D}$ with the colour sets of the places;

- one column $M^{0}$ with an initial marking of the places.

Table 2 shows the guards and the initial marking but does not show the colour sets, nor the time increments, nor the shaded border transitions $t_{a}, \ldots, t_{f}$, nor the constituents of the fused places $s_{1}, s_{3}$ and $s_{4}$ (see Section 4.2).

An event at a transition $t$ is determined by a binding, $e$, that assigns the individual variables of $t$ to values such that the guard of $t$ is satisfied. Its effect to a place $s$ is given by applying $e$ to the corresponding entry of the incidence matrix,

$$
\Delta M_{s}=C_{s}^{t} \odot e
$$

Keep in mind that the guard of $t, \mathscr{G}_{t}$, is a Boolean coefficient that is common to all arc expressions around $t$; hence it is evaluated as part of $C_{s}^{t} \odot e$.

Event $e$ is enabled - may occur - at state $M$ if (see equations (4)-(6))

$$
M-{ }^{t} \odot e \geqslant 0
$$

This is the so-called 'weak enabling' which is derived from (2) through a series of abstractions.

A process $M_{1} \stackrel{P}{\longrightarrow} M_{2}$ leads from state $M^{1}$ to state $M^{2}$ through a partially ordered set of event occurrences, $P$. If we ignore the order of occurring we get a T-vector, $u$, of combinations of events - a T-marking - that is called the action performed by the process. The state difference is

$$
\Delta M=M^{2}-M^{1}=C \cdot u
$$


A non-trivial action $u$ is called a $c y c l e e^{9}$ iff $C \cdot u=0$. Every process performing a cycle leads from a state to the same state again.

If $\delta$ is a distribution whose domain is the colour set of a place $s, \delta$ can be applied to all entries of row $C^{s}$ of the incidence matrix; it is a distribution of the place $s$. Now let $V$ be an S-vector such that for each place $s$ the component $V_{s}$ is a combination of distributions of $s$ and all the $V_{s}$ have the same range. Then the transposed incidence matrix $C^{\top}$ as well as every transposed marking $M^{\top}$ can be multiplied by the one column matrix $V$.

$$
\begin{aligned}
\left(C^{\top} \cdot V\right)_{t} & =\sum_{s \in S} C_{s}^{t} \odot V_{s} \\
M^{\top} \cdot V & =\sum_{s \in S} M_{s} \odot V_{s}
\end{aligned}
$$

Obviously, any such S-vector $V$ defines a state quantity of the net system represented by $C$. The dot product $M^{\top} \cdot V$ is the value of $V$ at state $M$, and soon (Section 6.2, formula (38)) it will turn out that $C^{\top} \cdot V$ is the gradient of $V$, the T-vector of partial derivatives of $V$ with respect to the transitions. If the gradient is the zero vector, $V$ is constant. ${ }^{10}$

A state quantity $V$ defined by a vector of (combinations of) distributions is linear in the markings. For any coefficient $k$ and two markings $M^{a}=\left(M_{1}^{a}, \ldots, M_{m}^{a}\right)$ and $M^{b}=\left(M_{1}^{b}, \ldots, M_{m}^{b}\right)$,

$$
\begin{aligned}
V\left(M^{a}+M^{b}\right) & =V\left(M^{a}\right)+V\left(M^{b}\right) \\
V\left(k \cdot M^{a}\right) & =k \cdot V\left(M^{a}\right)
\end{aligned}
$$

Conversely, any linear state quantity $V$ can be represented by an S-vector of distributions, $\left(V_{1}, \ldots, V_{m}\right)$. Because of linearity, we have for any marking $M$

$$
V(M)=V\left(M_{1}, \ldots, M_{m}\right)=V\left(\sum_{s=1}^{m}\left(0, \ldots, M_{s}, \ldots, 0\right)\right)=\sum_{s=1}^{m} V\left(0, \ldots, M_{s}, \ldots, 0\right)
$$

Hence we set

$$
V_{s}=\left\{p_{s} \mapsto V\left(0, \ldots, p_{s}, \ldots, 0\right)\right\}
$$

where $p_{s}$ is a generic pattern for the colour set of place $s$.

\section{Characteristic Functions}

\subsection{Dynamics}

Two systems of the same type may be quite different dynamically. They are equal if all dependent variables are in pairs represented by the same functions of the coordinates. To tell them apart, however, it is sufficient to consider one particular function.

Fundamental Law of Dynamics. For any family of systems of the same type with $n$ degrees of freedom there exists at least one way to select $n+1$ variables $X_{1}, \ldots, X_{n}, Y$ such that the $X_{i}$ form a coordinate system and the function $Y=Y\left(X_{1}, \ldots, X_{n}\right)$ identifies each individual system of the family. $Y=Y\left(X_{1}, \ldots, X_{n}\right)$ is called the characteristic function of the individual system associated with the coordinate system $X_{1}, \ldots, X_{n}$.

Note that characteristic function and coordinate system belong together. In general there are different characteristic functions associated with different coordinate systems.

For every system there is a coordinate system for which energy is the characteristic function. The two

\footnotetext{
9 Also known as a T-invariant.
}

10 Also known as an S-invariant. 
systems of Fig. 1, for example, are characterised by the quite different energy functions

$$
\begin{array}{ll}
\Sigma^{a}: E=K x+E_{0}^{a} & (K=m g) \\
\Sigma^{b}: E=\frac{\kappa}{2} x^{2}+E_{0}^{b} & (x<0)
\end{array}
$$

$K$ (mass $m$ times gravitational constant $g$ ) denotes the attractive gravitational force, $-\kappa x$ the restoring force exerted by the spring against the displacement $x$.

It is always possible to select coordinates $X_{1}, \ldots, X_{n}$ in such a way that energy $E$ is the characteristic function and is homogeneous of degree 1 in the coordinates.

$$
E=\sum_{j=1}^{n} \xi_{j} X_{j}, \quad \xi_{j}=\frac{\partial E\left(X_{1}, \ldots, X_{n}\right)}{\partial X_{j}}
$$

The variables $E, X_{1}, \ldots, X_{n}$ are called extensive, and the energy conjugates $\xi_{j}$ are called intensive. The extensive variables are the natural exchange variables. Examples: energy (including mass as rest energy), linear momentum, angular momentum, electrical charge, entropy, particle numbers, volume. The dynamical variables - extensive and intensive ones - have fixed zeros; they are 'absolute' like 'absolute' temperature, pressure, charge, particle numbers.

\subsection{Net Theory}

Net theory answers the question whether two systems are equal by stating the extensionality principle (see equation (2)). This means that the en-ability $\mathscr{E}$ of a state $c$, the set of events enabled at $c$, is a characteristic function of net systems - the only one known.

$$
e \in \mathscr{E}(c) \Longleftrightarrow \cdot e-e^{\bullet} \subseteq c \wedge e^{\bullet}-\cdot e \subseteq \bar{c}
$$

Hence the extensionality principle may also be read in the following way.

Fundamental Law of Net Theory. Two net systems $\Sigma^{a}$ and $\Sigma^{b}$ of the same type with $n$ places are equal if their en-ability functions $\mathscr{E}^{a}=\mathscr{E}^{a}\left(s_{1}^{a}, \ldots, s_{n}^{a}\right)$ and $\mathscr{E}^{b}=\mathscr{E}^{b}\left(s_{1}^{b}, \ldots, s_{n}^{b}\right)$, are equal.

In higher-level net models classes of conditions are abstracted to places and classes of events are abstracted to transitions. A condition at place $s$ is given by the presence of a piece - token - on $s$. An event at transition $t$ is given by a binding of the individual variables of $t$ satisfying the guard of $t$; enabling and effect are described in (13) and (14). This defines the system variable en-ability, $\mathscr{E}=\mathscr{E}(M)$, for markings $M . \mathscr{E}(M)$ gives for every event the multiplicity of its enabling in $M$.

\section{Composition of Systems}

\subsection{Dynamics}

The composition of two systems is defined by the addition of their - comparable - characteristic functions. For example, the composition of the two separate systems in Fig. 1 yields

$$
E=E^{a}+E^{b}=K x^{a}+\kappa \frac{x^{b^{2}}}{2}+E_{0}^{a}+E_{0}^{b}
$$

Through the coupling $x^{a}+x^{b}=$ const. $=0, x=x^{a}=-x^{b}$ (see Fig. 2) we get

$$
E=E^{a}+E^{b}=K x+\kappa \frac{x^{2}}{2}+E_{0} \quad(x<0)
$$

In the total system, processes of the form

$$
\begin{gathered}
X_{1}^{a}+X_{1}^{b}=X_{1}=\text { const., } ., X_{j}^{a}+X_{j}^{b}=X_{j}=\text { const., } \\
X_{j+1}^{a}=\text { const., ., } X_{m}^{a}=\text { const., } \\
X_{j+1}^{b}=\text { const., ., } X_{n}^{b}=\text { const. }
\end{gathered}
$$



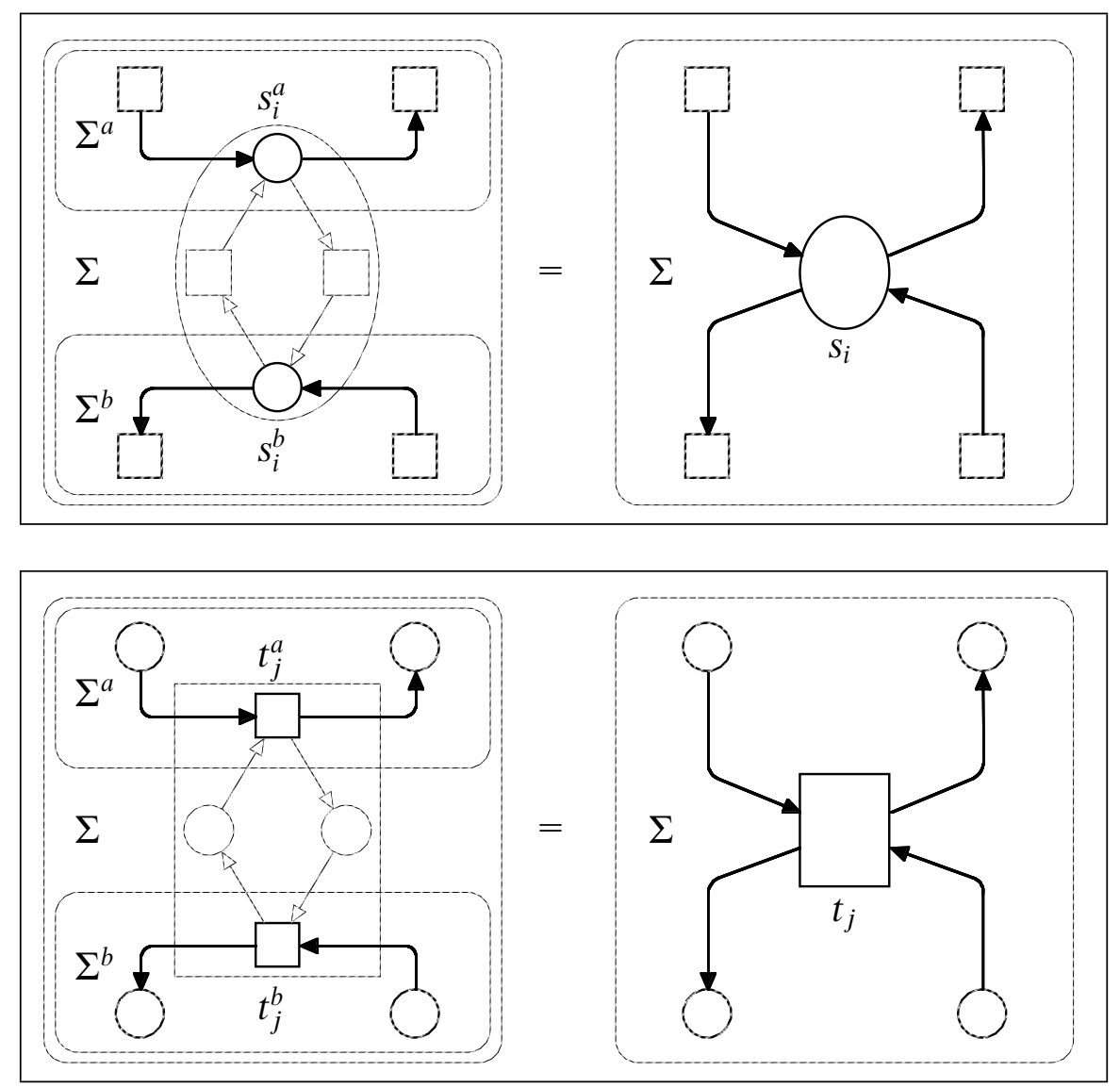

Fig. 4. Coupling of net systems by place fusion and by transition fusion.

are called exchange processes. The (extensive or intensive) variables $X_{k}^{\sigma}$ form a complete coordinate system. With respect to the exchange process the variables $X_{1}, \ldots, X_{j}$ are called exchange variables and their conjugates $\xi_{1}, \ldots, \xi_{j}$ contact variables. The extensive variables are the preferred, 'natural' exchange variables yet not the only ones. In a stable equilibrium the contact variables have the same value.

For the exchange process $E^{a}+E^{b}=E=$ const. in Fig. 2 and equation (25), energy is the exchange variable and the conjugate force $\frac{\partial E}{\partial x}$ is the contact variable. In stable equilibrium, the two forces $\frac{\partial E_{a}}{\partial x}=K$ and $\frac{\partial E_{b}}{\partial x}=-\kappa x$ permanently have the same value: $x=x_{0}=x(t=0)=-\frac{K}{\kappa}$; the system is at a standstill.

\subsection{Net Theory}

The composition of two net systems is defined by the addition (disjoint union) of their nets (graphs and matrices). Coupling can be achieved in two ways: through the fusion of places and the fusion of transitions. Figure 4 suggests that place fusion may be viewed as an abstraction from the 'free exchange' of tokens between comparable places (analogous to equation (1)), and transition fusion as an abstraction from the 'synchronous communication' between comparable transitions [ChH94].

The production scheme in Fig. 3 can be seen as the result of coupling the production with an administration; the places $s_{1}$ and $s_{3}$ are the result of fusing the respective border places, $s_{i}$ and $s_{i}^{\prime}(i=1,3,4)$. The system may be closed by fusing the shaded border transitions with corresponding complements in an environment. 


\section{Time}

\subsection{Dynamics}

A process of a physical system appears as a continuous sequence of states. The variables are functions of the independent parameter time. The characteristic function of a system (see Section 3.1) becomes $Y=Y\left(X_{1}, \ldots, X_{n}, t\right)$.

The coupled mass-spring system in Fig. 2 reacts to a displacement from its equilibrium with oscillating. We describe the states - the values of the single coordinate $x$ - as a function of time, $x=x(t)$. The family of such functions, depending on the initial displacement, is determined by integrating the differential equation

$$
\frac{d \mathbf{P}}{d t}=m \frac{d^{2} x}{d t^{2}}=K-\kappa x \quad(x<0, K=-m g)
$$

We get

$$
x(t)=\hat{x} \cos \omega t+x_{0} \quad\left(x<0, x_{0}=\frac{-m g}{\kappa}, \hat{x}=x(t=0)-x_{0}, \omega=\sqrt{\frac{\kappa}{m}}\right)
$$

\subsection{Net Theory}

In net theory, a process $M_{1} \stackrel{P}{\longrightarrow} M_{2}$ is a set of event occurrences, partially ordered by a causal relation earlier, connecting two states. Every occurrence of an event determines a point in time; conditions hold for a duration greater than zero. The duration of a process is the maximum length of the maximal condition sequences. The total temporal order of event occurrence is stricter than the partial causal order earlier. A state can be determined only at times when no event occurs.

Let us now try to determine the course of a process in time explicitly. The occurrence of an event affects the enabling of other events through the starting and finishing of condition holdings. We now say that every event $e=\Delta t$ determines a minimal duration for all conditions that begin to hold with an occurrence of $e$. And the point in time of its occurrence is the earliest possible such that no condition finishes to hold before its minimal duration is over.

In timed models of Design/CPN [CPN96], the tokens carry time stamps that hold the earliest point in (simulation) time at which the token may be consumed by an event occurrence. The value of the time stamp of a token created is determined as a function of the point in time of the creation and an increment connected with the token in the corresponding arc expression; a time region of a transition may hold an increment that is common to all the tokens created. The point in time of a transition occurrence then is the maximal time stamp value of all tokens being consumed.

Time does not play a role for the rest of this paper. The time increments of the production scheme in Fig. 3 will be ignored.

\section{Forms of State Quantities}

\subsection{Dynamics}

Energy appears in different forms, and it can be converted from one form into another.

\section{Examples.}

(a) Energy of motion: the change of energy $d E$ appears together with a change of momentum; $d \boldsymbol{P} . \boldsymbol{v}=\boldsymbol{v}(\boldsymbol{P})$ denotes the velocity of the body in motion.

$$
d E=\boldsymbol{v} d \boldsymbol{P}=v_{x} d P_{x}+v_{y} d P_{y}+v_{z} d P_{z}
$$

(b) Energy of stress: the change of energy $d E$ appears together with a displacement $d x$ and a change $d V$ of a volume $V$ (component of force $K_{x}=\boldsymbol{K}(x)$, pressure $p=p(V)$ ).

$$
d E=K_{x} d x \quad \text { respectively } \quad d E=p d V
$$


(c) Thermal energy: the change of energy $d E$ appears together with a change of $d S$ of entropy (absolute temperature $T=T(S))$.

$$
d E=T d S
$$

An energy form is defined in terms of an extensive coordinate $X: d E=\xi d X$, where the intensive variable $\xi$ may depend on $X$ (and other independent variables).

In general, if $X_{1}, \ldots, X_{n}$ are coordinates of a system with $n$ degrees of freedom, any infinitesimal change of a dependent variable $Y=Y\left(X_{1}, \ldots, X_{n}\right)$ is a linear combination of $n$ different forms in which $Y$ appears in terms of the different coordinates.

$$
d Y=\frac{\partial Y}{\partial X_{1}} d X_{1}+\ldots+\frac{\partial Y}{\partial X_{n}} d X_{n}
$$

\subsection{Net Theory}

Since the places are the coordinates of a net system, we now want to study in which form a state quantity of a net system may appear at the different places.

There are no infinitesimal changes in a net system. The 'smallest' changes are single events. Let $\Delta t$ be an event at transition $t$ given by a binding $e$ for $t$. The effect of an occurrence of $\Delta t$ on a single place $s$ is $C_{s}^{t} \odot e$; see (13). We now set out to interpret, and write, $C_{s}^{t}$ as the partial derivative of coordinate $s$ with respect to transition $t$ and rewrite (13) as

$$
\Delta s=\Delta M_{s}=C_{s}^{t} \odot e \cong \frac{\partial s}{\partial t} \Delta t
$$

The symbol $\cong$ is to denote an analogy whose power we have yet to explore. There is no claim that the use of some notation of calculus may lead to more than certain similarities. In fact the similarities are limited to linear quantities of net systems (see (18) and (20)). In this restricted area, however, they seem quite striking and worth exploring. And they are not purely accidental. At the end of our exploration, when we have seen more of the purpose and power of this analogy, the proper foundation in calculus will be shown (Section 7.3).

To begin with, let us have a look at the effect of an event $\Delta t$ on a linear state quantity given by an S-vector of distributions.

$$
\Delta V=V(\Delta M)=\sum_{s} V_{s}(\Delta s)=\sum_{s} V_{s}\left(\frac{\partial s}{\partial t} \Delta t\right)
$$

We interpret the distributions $V_{s}$ as partial derivatives of the variable $V$ with respect to the coordinates $s$,

$$
\frac{\partial V}{\partial s} \cong V_{s}
$$

and get

$$
\Delta V=\frac{\partial V}{\partial s_{1}} \Delta s_{1}+\ldots+\frac{\partial V}{\partial s_{n}} \Delta s_{n}
$$

Matching this to (29) we call the distribution $V_{s}$ the form in which the state quantity $V$ appears at the place $s$. $V_{s}$ shows in which way the pieces on place $s$ contribute to the value of quantity $V$.

If the two derivatives make sense or, at least, are consistent so far there should also be the partial derivative of $V$ with respect to transition $t$ such that

$$
\begin{aligned}
& \Delta V=\frac{\partial V}{\partial t} \Delta t \\
& \frac{\partial V}{\partial t}=\sum_{s} \frac{\partial V}{\partial s} \frac{\partial s}{\partial t}
\end{aligned}
$$

The following two parallel derivations for the effect $\Delta V$ of an event $e=\Delta t$ on $V$ demonstrate that this is 
Table 3. Three linear variables of the production scheme given by their forms

\begin{tabular}{|c|c|c|c|c|c|c|c|c|}
\hline & \multicolumn{2}{|c|}{ load } & \multicolumn{3}{|c|}{ recipe } & \multicolumn{3}{|c|}{ count } \\
\hline$s_{0}$ & $(x, c)$ & $\mapsto c^{6} x$ & - & $\mapsto$ & $\mathbf{0}$ & $(-, c)$ & $\mapsto$ & $>0] \cdot c^{6}()$ \\
\hline$s_{1}$ & $\left(x, c,_{-},,_{-}\right)$ & $\mapsto c^{6} x$ & $\left({ }_{-},{ }_{-}, r, r^{\prime}\right)$ & $\mapsto$ & $1^{`}\left(\operatorname{rev}\left(r^{\prime}\right)^{\wedge} r\right)$ & $\left({ }_{-}, c,,_{-},{ }_{-}\right)$ & $\mapsto$ & $>0] \cdot c^{6}()$ \\
\hline$s_{2}$ & $\left(x, c,,_{-},,_{-}\right.$ & $\mapsto c^{6} x$ & $\left({ }_{-},{ }_{-}, d, r, r^{\prime}\right.$ & $\mapsto 1$ & $\left(\operatorname{rev}\left(d:: r^{\prime}\right)^{\wedge \wedge} r\right)$ & $\left({ }_{-}, c,,_{-},,_{-}\right.$ & )$\mapsto$ & $>0] \cdot c^{6}()$ \\
\hline$s_{3}$ & $x$ & $\mapsto 1^{\prime} x$ & - & $\mapsto$ & 0 & - & $\mapsto$ & $1^{\circ}()$ \\
\hline$s_{4}$ & $x$ & $\mapsto \mathbf{0}$ & $r$ & $\mapsto$ & $1^{\prime} r$ & - & $\mapsto$ & 0 \\
\hline$s_{5}$ & $\left(x, c^{\prime},{ }_{-}\right)$ & $\mapsto c^{\prime \prime} x$ & - & $\mapsto$ & $\mathbf{0}$ & $\left({ }_{-}, c^{\prime},{ }_{-}\right)$ & $\mapsto$ & $\geqslant 0] \cdot c^{\prime \prime}()$ \\
\hline$s_{6}$ & $(x, c)$ & $\mapsto c^{6} x$ & - & $\mapsto$ & $\mathbf{0}$ & $(-, c)$ & $\mapsto$ & $>0] \cdot c^{6}()$ \\
\hline$s_{7}$ & - & $\mapsto \mathbf{0}$ & $(-, r, n)$ & $\mapsto$ & $-n^{6} r$ & - & $\mapsto$ & 0 \\
\hline$s_{8}$ & - & $\mapsto \mathbf{0}$ & - & $\mapsto$ & $\mathbf{0}$ & - & $\mapsto$ & $\mathbf{0}$ \\
\hline
\end{tabular}

indeed the case.

$$
\begin{aligned}
& \Delta V=\sum_{s} V_{s}\left(C_{s}^{t} \odot e\right) \stackrel{!}{=} \sum_{s}\left(C_{s}^{t} \odot V_{s}\right) \odot e=\left(\sum_{s} C_{s}^{t} \odot V_{s}\right) \odot e=\left(C^{t^{\top}} \cdot V\right) \odot e \\
& \Delta V=\sum_{s} \frac{\partial V}{\partial s}\left(\frac{\partial s}{\partial t} \Delta t\right)=\sum_{s}\left(\frac{\partial V}{\partial s} \frac{\partial s}{\partial t}\right) \Delta t=\left(\sum_{s} \frac{\partial V}{\partial s} \frac{\partial s}{\partial t}\right) \Delta t=\frac{\partial V}{\partial t} \Delta t
\end{aligned}
$$

The essential step in (36) is due to the fact that distributions and substitutions commute. Equation (36) also shows how to compute $\frac{\partial V}{\partial t}$ as the dot product of $C^{t}$ and $V$. Consequently, the matrix product of $C^{\top}$ with the one column matrix $V$ yields the vector of partial derivatives with respect to the transitions, the gradient of $V, \nabla V$.

$$
\nabla V=\left(\ldots, \frac{\partial V}{\partial t}, \ldots\right)=\left(C^{\top} \cdot V\right)
$$

If the gradient is the zero vector, $V$ is constant, $Y=$ const. $=Y\left(M^{0}\right)$; it is what in the literature is known as an $S$-invariant or a place semi-flow.

If $V$ can be split into two non-trivial, non-negative variables $U$ and $W$ such that $V=U-W$, i.e if $V$ has mixed signs, and $V$ is constant, $U$ and $W$ are, up to a constant, copies of each other: $U=W+V\left(M^{0}\right)$. The notion copy does not appear in the dynamics of continuous systems but is of fundamental importance in digital information processing. Digital data are distinguished from analogue data by the fact that exact copies can be made of digital data arbitrarily often: $\operatorname{copy}(\operatorname{copy}(\ldots(\operatorname{copy}(d)) \ldots))=d$.

It seems high time for some examples. Table 3 shows three state quantities of the production scheme (Fig. 3) that are given by the forms in which they appear at the different places. The quantity load is the actual workload of the system, the sum of all batches currently being present somewhere, in one of the five following forms:

$$
\begin{aligned}
& \operatorname{load}(M)=\quad M\left(s_{0}\right) \odot\{(x, c) \mapsto c \cdot x\} \quad \text { order waiting } \\
& +M\left(s_{1}\right) \odot\left\{\left(x, c,_{-,}\right) \mapsto c \cdot x\right\} \quad \text { batch (remainder) being started } \\
& +M\left(s_{2}\right) \odot\left\{\left(x, c,,_{-,},-\right) \mapsto c \cdot x\right\} \quad \text { batch at a station } \\
& +M\left(s_{3}\right) \odot\{x \mapsto 1 \cdot x\} \quad \text { single unit done } \\
& +M\left(s_{5}\right) \odot\left\{\left(x, c,,_{-}\right) \mapsto c \cdot x\right\} \quad \text { units on shelf } \\
& +M\left(s_{6}\right) \odot\{(x, c) \mapsto c \cdot x\} \quad \text { product ready for delivery }
\end{aligned}
$$

Similarly the quantity recipe gives the recipes for those batches currently being processed. Count is an auxiliary quantity that looks at the meaningful, i.e. non-negative multiplicities of the batches. It can be used to prove that the condition $c>0$ in the guard of $t_{4}$ that comes in handy for the reduction of partial derivatives at $t_{4}$ is redundant.

Table 4 shows the result of applying each form of load to all entries of the corresponding row of the incidence matrix. In addition, the bottom row shows the resulting column sums: the gradient of quantity load. Now note that at $t_{6}$, the guard says $c^{\prime}=c$. Hence the derivative is zero. At $t_{4}$, the guard says $c>0$. Formally, 
Table 4. Gradient of the workload, $C^{\top} \cdot$ load

\begin{tabular}{|c|c|c|c|c|c|c|}
\hline & $t_{1}$ & $t_{2}$ & $t_{3}$ & $t_{4}$ & $t_{5}$ & $t_{6}$ \\
\hline$\Gamma$ & $\begin{array}{l}{[c>0,} \\
r \neq[]]\end{array}$ & & {$[r \neq[]]$} & $\begin{array}{c}{[c>0,} \\
r=[]]\end{array}$ & {$\left[c^{\prime}=c\right]$} & \\
\hline$s_{0}$ & $-c \cdot x$ & & & & & \\
\hline$s_{1}$ & $c \cdot x$ & $-c \cdot x$ & $c \cdot x$ & {$[c>1] \cdot(c-1) \cdot x$} & & \\
\hline$s_{2}$ & & $c \cdot x$ & $-c \cdot x$ & $-c \cdot x$ & & \\
\hline $\begin{array}{l}s_{3} \\
s_{4}\end{array}$ & & & & $x$ & $-x$ & \\
\hline $\begin{array}{l}s_{5} \\
s_{6} \\
s_{7}\end{array}$ & $0 \cdot x$ & & & & $\begin{array}{r}-c^{\prime} \cdot x \\
+\left(c^{\prime}+1\right) \cdot x\end{array}$ & $-c^{\prime} \cdot x$ \\
\hline$s_{8}$ & & & & & & $c \cdot x$ \\
\hline Dload & $\mathbf{0}$ & $\mathbf{0}$ & $\mathbf{0}$ & $\begin{array}{r}x-c \cdot x \\
-[c>1] \cdot(x-c \cdot x)\end{array}$ & $\mathbf{0}$ & $\begin{array}{l}-c^{\prime} \cdot x \\
+c \cdot x\end{array}$ \\
\hline
\end{tabular}

Table 5. Gradient of the variable recipe

\begin{tabular}{|c|c|c|c|c|c|c|}
\hline & $t_{1}$ & $t_{2}$ & $t_{3}$ & $t_{4}$ & $t_{5}$ & $t_{6}$ \\
\hline$\Gamma$ & $\begin{array}{l}{[c>0,} \\
r \neq[]]\end{array}$ & & {$[r \neq[]]$} & $\begin{array}{c}{[c>0,} \\
r=[]]\end{array}$ & & {$[c \geqslant 0]$} \\
\hline Drecipe & $\begin{array}{r}(\operatorname{rev}[])^{\wedge} r \\
-r\end{array}$ & $\begin{array}{l}-\operatorname{rev}\left(r^{\prime}\right)^{\wedge}(d:: r) \\
\quad+\operatorname{rev}\left(d:: r^{\prime}\right)^{\wedge}{ }^{\wedge}\end{array}$ & 0 & $\begin{array}{r}{[c>1] \cdot\left(\operatorname{rev}([])^{\wedge} \wedge \operatorname{rev}\left(d:: r^{\prime}\right)\right)} \\
-\operatorname{rev}\left(d:: r^{\prime}\right)^{\wedge} r \\
+[c=1] \cdot \operatorname{rev}\left(d:: r^{\prime}\right)\end{array}$ & 0 & 0 \\
\hline
\end{tabular}

a guard is a Boolean coefficient that is common to all arc expressions. We can multiply the derivative at $t_{4}$ with $[c>0]$. Since $[c>0]=[c=1]+[c>1]$ and $[c>0] \cdot[c>1]=[c>1]$, we get

$$
\begin{array}{r}
{[c>0] \cdot(x-c \cdot x)} \\
-[c>0] \cdot[c>1] \cdot(x-c \cdot x)
\end{array}=\begin{array}{r}
{[c>0] \cdot(x-c \cdot x)} \\
-[c>1] \cdot(x-c \cdot x)
\end{array}=[c=1] \cdot(x-c \cdot x)=\mathbf{0}
$$

The gradient of quantity load is zero; the value load is constant. The value of load for some marking $M$ is the dot product of $M$ and load.

$$
\begin{aligned}
\operatorname{load}(M)=\operatorname{load}\left(M^{0}\right) & =((\underline{X}, 3)+(\underline{Y}, 2)) \odot\{(x, c) \mapsto c \cdot x\}+\ldots 0 \ldots \\
& =3 \cdot \underline{X}+2 \cdot \underline{Y}
\end{aligned}
$$

In the same way the gradient of the quantity recipe is calculated. The result is shown in Table 5. Since the calculation was done with the help of the experimental software package rather than with paper and pencil, the details are given in Section A.4 of the Appendix.

The partial derivatives with respect to transitions $t_{1}$ and $t_{2}$ reduce to zero due to the properties of the list operator rev. And at $t_{4}$, this time both $c>0$ and $r=[$ ] of the guard are used to reduce the partial derivative to zero. Hence we have

$$
\operatorname{recipe}(M)=\text { const } .=\operatorname{recipe}\left(M^{0}\right)=M_{0}^{\top} \cdot \text { recipe }=\mathbf{0}
$$

In contrast to load the forms of recipe have mixed signs.

$$
\begin{aligned}
& \operatorname{recipe}(M)=\operatorname{recipe}^{(\geqslant 0)}\left(s_{1}, s_{2}, s_{4}\right)-\operatorname{recipe}^{(\leqslant 0)}\left(s_{7}\right)=\mathbf{0} \\
& \operatorname{recipe}^{(\geqslant 0)}(M)=\operatorname{recipe}^{(\leqslant 0)}(M)
\end{aligned}
$$


A copy of the recipe of every batch being processed, distributed over the places $s_{1}, s_{2}$ and $s_{4}$, is kept on place S7.

\section{Process Quantities}

\subsection{Dynamics}

Quantities like work or heat belong to processes rather than states. If, for example, the trajectory of a point mass $m$ is $P=\vec{r}(t)=(x(t), y(t), z(t))\left(t_{a} \leqslant t_{e}\right)$ and the force acting on $m$ is the vector field $\vec{F}(x, y, z)$, the work done by $\vec{F}$ in the course of $P$ is the line integral

$$
W=\int_{P} \vec{F} d \vec{r}=\int_{t_{a}}^{t_{b}}\left(\vec{F}(\vec{r}(t)) \cdot \frac{d \vec{r}}{d t}\right) d t
$$

If the work depends on the initial and terminal states only rather than on the course of the process itself, the force is conservative (without friction, in particular). In this case there exists a state quantity $U=U(x, y, z)$ such that $\vec{F}$ is the negative gradient field of the variable $U, \vec{F}=-\left(\frac{\partial U}{\partial x}, \frac{\partial U}{\partial y}, \frac{\partial U}{\partial z}\right)=-\nabla U . U$ is unique up to a constant $U_{0}$ and is called the potential function of $\vec{F}$. The - sign is to ensure that if $\vec{F}$ is the gravitational force then $U$ is the potential energy. $W=U\left(s_{2}\right)-U\left(s_{1}\right)$ is the work done against $\vec{F}$ by going from state (position) $\vec{r}_{1}$ to $\vec{r}_{2}$.

\subsection{Net Theory}

In net systems a process $M_{1} \stackrel{P}{\longrightarrow} M_{2}$ is a partially ordered finite set of event occurrences that lead from the initial to the terminal state. Let $\left\{\Delta t_{i}\right\}_{0 \leqslant i \leqslant n}$ be a 'firing sequence', i.e. an enumeration of the event occurrences of $P$ that is compatible with the partial order $\prec$ (earlier),

$$
\Delta t_{i} \prec \Delta t_{k} \Rightarrow i<k
$$

Index $i$ corresponds to the parameter time; the transition occurrences are the time points.

Let $\mathscr{F}=(\ldots, \mathscr{F} t, \ldots)$ be a $\mathrm{T}$-vector of expressions denoting combinations of pieces of the same type such that for each $t$ the individual variables of $\mathscr{F}_{t}$ belong to $t$. The T-vector $\mathscr{F}$ uniquely determines a linear process quantity $W$ by virtue of

$$
W(P)=\sum_{i} \mathscr{F}_{t} \odot \Delta t_{i}=\mathscr{F}^{\top} \cdot P
$$

which we now interpret, in analogy to (39), as the line integral of the field $\mathscr{F}$ along the process $P$.

$$
W(P)=\sum_{i} \mathscr{F}_{t} \odot \Delta t_{i} \cong \int_{P} \mathscr{F} d T
$$

In analogy to the forms in which a state quantity appears at the places, we call the components $\mathscr{F}_{t}$ the forms of the process quantity $W$ at the transitions $t$.

Example. The gradient of a linear state quantity $V$ (see equation (38)) gives the forms in which the work on $V$ is done at the transitions during a process.

Non-Example. In general, in the presence of concurrency, the duration of a process cannot be determined by a field.

The following facts on the relationship between derivatives and integrals will provide a kind of final test for the consistency of the notions and notation transferred from physics to net theory.

Theorem 7.1. If $\mathscr{F}=\nabla V$ then $\int_{P} \mathscr{F} d T$ is the change $\Delta V=V\left(M_{2}\right)-V\left(M_{1}\right)$ effected by some process $M_{1} \stackrel{P}{\longrightarrow} M_{2}$. 
Proof. Immediate consequence of (36).

Theorem 7.2. The derivative of a distribution $\left\{\left(x_{1}, \ldots, x_{n}\right) \mapsto u\right\}$ is its image expression $u$, and the indefinite integral of an expression $u$ is the distribution $\left\{\left(x_{1}, \ldots, x_{n}\right) \mapsto u\right\}$ where the $x_{i}$ are the free individual variables of $u$.

Proof. Consider the following trivial net:

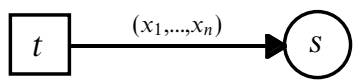

Let $u$ be an arbitrary expression denoting a combination of pieces in terms of the individual variables $x_{1}, \ldots, x_{n}$. Let $V$ be the linear quantity defined by the corresponding distribution $\delta=\left\{\left(x_{1}, \ldots, x_{n}\right) \mapsto u\right\}$. Let $p$ be an arbitrary combination of pieces on $s$ and, with pattern matching $\equiv, \hat{p}=\left(x_{1}, \ldots, x_{n}\right) \equiv p$ the corresponding combination of substitutions. Then according to our definitions

$$
\begin{gathered}
\frac{d V}{d t}=\left(x_{1}, \ldots, x_{n}\right) \odot \delta=u \\
\int_{0}^{p} u d t=u \odot \hat{p}=p \odot \delta=V(p)
\end{gathered}
$$

Theorem 7.3. For a field $\mathscr{F}$ there exists a potential function, a state quantity $U$ such that $\mathscr{F}=-\nabla U$, iff for any two processes $P$ and $P^{\prime}$ connecting the same pair of states the value of the line integral of $\mathscr{F}$ is the same:

$$
M_{1} \underset{P^{\prime}}{\stackrel{P}{\longrightarrow}} M_{2} \Rightarrow \int_{P} \mathscr{F} d T=\int_{P^{\prime}} \mathscr{F} d T
$$

or, equivalently, iff for any cyclic process $P$ the integral equals $\mathbf{0}$ :

$$
M \stackrel{P}{\longrightarrow} M \Rightarrow \oint_{P} \mathscr{F} d T=0
$$

Proof.

(a) Assume there is a state quantity $U$ with $\mathscr{F}=-\nabla U$ and let $P, P^{\prime}$ be such that $M_{1} \stackrel{P}{\longrightarrow} M_{2}$ and $M_{1} \stackrel{P^{\prime}}{\longrightarrow} M_{2}$. Then $\int_{P} \mathscr{F} d T=-U\left(M_{2}-M_{1}\right)=\int_{P^{\prime}} \mathscr{F} d T$.

(b) Assume that $\int_{P} \mathscr{F} d T=\int_{P^{\prime}} \mathscr{F} d T$ for any $P, P^{\prime}$ with $M_{1} \stackrel{P}{\longrightarrow} M_{2}$ and $M_{1} \stackrel{P^{\prime}}{\longrightarrow} M_{2}$. Let $U_{0}=U\left(M_{0}\right)=0$ for the initial marking $M_{0}$. For any reachable marking $M$ there is a process $P$ such that $M_{0} \stackrel{P}{\longrightarrow} M$. Set $U(M)=-\int_{P} \mathscr{F} d T ; U$ is a proper mapping since its value does not depend on the path leading from $M_{0}$ to $M$.

$U$ is linear and can be presented as an S-vector of distributions $\left(\ldots, U_{s}=\frac{\partial U}{\partial s}, \ldots\right)$ (see (20)). For any event $e=\Delta t$

$$
-\mathscr{F}_{t} \odot e=U(\Delta M)=\sum_{s} U_{s}\left(C_{s}^{t} \odot e\right)=\left(\sum_{s} \frac{\partial U}{\partial s} \frac{\partial s}{\partial t}\right) \odot e=\frac{\partial U}{\partial t} \odot e
$$

Hence $\mathscr{F}_{t}=-\frac{\partial U}{\partial t}, \mathscr{F}=-\nabla U$. 


\section{An Example}

For the production scheme in Fig. 3, let some production costs be given in units $\underline{u}$ per substance and per transition by the T-vector

$$
\cos t=\begin{array}{c|c} 
& \\
\hline t_{1} & 0^{c} \underline{u} \\
t_{2} & K_{2}(x, d)^{c} \underline{u} \\
t_{3} & 0^{c} \underline{u} \\
t_{4} & 0^{c} \underline{u} \\
t_{5} & K_{5}(\underline{x})^{6} \underline{u}
\end{array}
$$

where

$$
K_{2}(x, d)=\begin{array}{l|lll} 
& D_{1} & D_{2} & D_{3} \\
\hline X & 4.5 & 2.7 & 3.1 \\
Y & 3.0 & 2.0 & 5.0 \\
Z & 0.0 & 0.0 & 0.0
\end{array} \quad \text { and } \quad K_{5}(x)=\begin{array}{r|l} 
& \\
\hline X & 1.4 \\
Y & 1.0 \\
Z & 0.0
\end{array}
$$

The vector cost is the cost field of production. If, for example, $P$ is the process of producing three copies of item $X$, the costs made by $P$ are

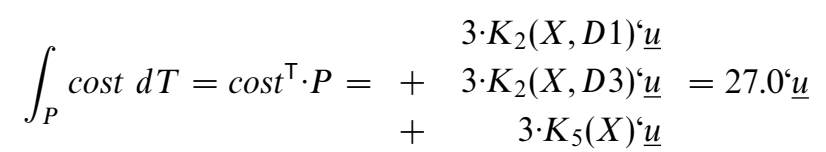

In this simple example, at any stage of production the remaining costs for processing the current work load can be predicted. There is a state quantity $\operatorname{COS} T$ such that for any process $M_{1} \stackrel{P}{\longrightarrow} M_{2}$, the costs of $P$, $\int_{P} \operatorname{cost} d T$, are $\operatorname{COS} T\left(M_{1}\right)-\operatorname{COS} T\left(M_{2}\right)$. $\operatorname{COS} T$ can be defined by its forms in the following way:

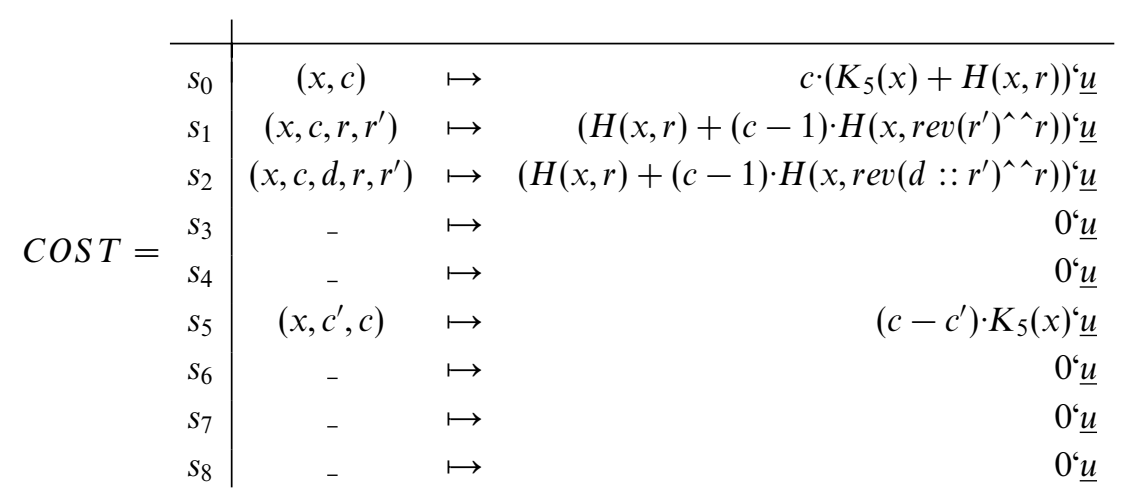

where $H(x, r)$ is an auxiliary function that for substance $x$ and remainder $r$ of its recipe determines the costs at transition $t_{2}$ :

$$
H=\left\{(x,[]) \mapsto 0\left|(x,[d]) \mapsto K_{2}(x, d)\right|\left(x, r^{\wedge} r^{\prime}\right) \mapsto H(x, r)+H\left(x, r^{\prime}\right)\right\}
$$

The quantity $\operatorname{COST}$ is a potential function for the cost field cost. The negative of the product of the incidence matrix $C$ with $\operatorname{COST}$ reduces to cost (by means of the guards of $t_{1}$ and $t_{4}$ ); cost is the negative gradient field of $\operatorname{COST},-\nabla \operatorname{COS} T=$ cost. The calculation shown in Table 6 was done by means of the software tool; details are given in the Appendix (see A.5).

If we extend the system by the shaded border transitions $t_{a}$ : 'accept new order' and $t_{b}$ : 'deliver product', there are cyclic processes with non-zero costs and hence no potential function can exist for the cost field (Theorem 7.3). 
Table 6. Calculating of a negative gradient field.

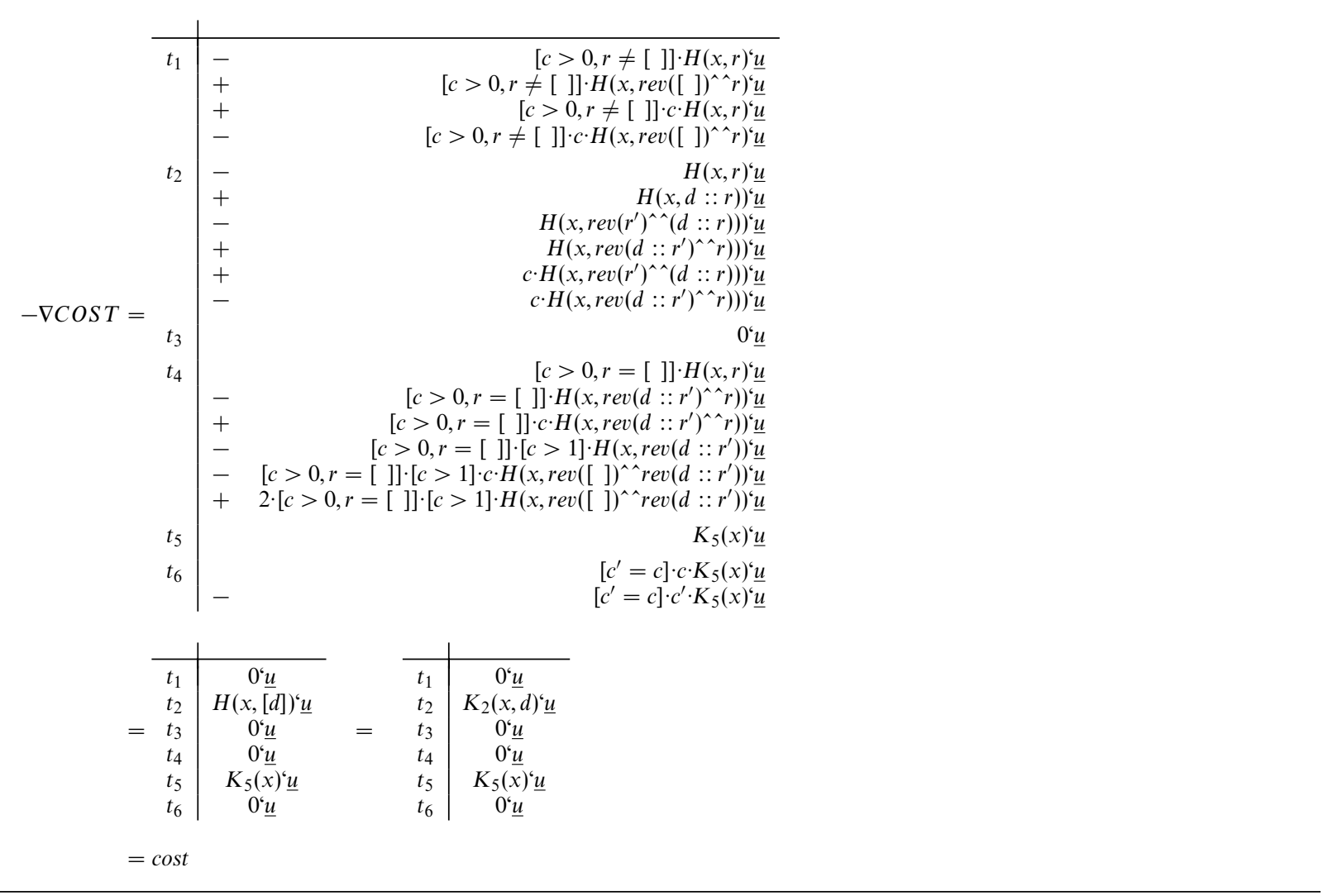

\subsection{The Incidence Matrix and Partial Derivatives}

Now that we have seen the extent to which the analogy between dynamics and net theory works, let us return to the question whether the similarities are purely accidental.

Assume that $C$ is the incidence matrix of a conventional Petri net without loops. Then the entries of $C$ are just integer numbers; the markings are place vectors of non-negative integers, and for every marking (state) $M=\left(s_{1}, \ldots, s_{n}\right)$ in the full reachability class of the initial marking $M^{0}$ there is a transition vector $u=\left(t_{1}, \ldots, t_{m}\right)$ of integers such that (see equation $\left.(15)\right)$

$$
M=M^{0}+C \cdot u=M^{0}+\sum_{1 \leqslant k \leqslant m} t_{k} \cdot C^{k}
$$

Note that we identify the transition $t_{k}$ and the variable $t_{k}$ telling the number of times $t_{k}$ occurs. If $t_{k}$ is negative, $M$ is in the past of the occurrences of $t_{k}$.

Let us now consider two generalisations which are purely mathematical rather than net theoretical. We ignore the restriction that the markings of places are non-negative, i.e. we ignore the enabling rule for transitions. And we allow arbitrary changes, in particular arbitrarily small changes, i.e we allow the $s_{i}$ and $t_{k}$ to be real numbers. Then

$$
M=\left(s_{1}, \ldots, s_{n}\right)=M\left(t_{1}, \ldots, t_{m}\right)=M^{0}+C \cdot u
$$

is a continuous vector function in $m$ variables. With equation (48) we get trivially:

$$
\frac{\partial M}{\partial t_{k}}=\left(\frac{\partial s_{1}}{\partial t_{k}}, \ldots, \frac{\partial s_{n}}{\partial t_{k}}\right)=C^{k} \quad \frac{\partial s_{i}}{\partial t_{k}}=C_{i}^{k}
$$


In other words, our notation making use of partial derivatives is firmly based on calculus. We have just lifted equation (50) and its implications to higher-level Petri nets. The similarities we observed between dynamics and net theory are a matter of course rather than accidental. They give additional evidence to the fact that Petri nets are adequate models of systems with combined continuous and discrete dynamics, that Petri nets are truely inter-disciplinary. And this, in turn, is a consequence of Petri's careful founding of net theory in accordance with the laws of physics (see [Pet62, Pet82], for example).

\section{Conservation Laws}

Laws of conservation and invariance are of fundamental importance for the understanding of systems and their embedding into environments. They can tell us that something is impossible - like a perpetuum mobile, for example, delivering more energy than it receives. They can help to adjust the border between a system and its environment, i.e. not to ignore certain phenomena if there is significant loss or gain of a quantity that should not change. The fact that the value of a particular variable, like load of the production scheme, remains constant within given system borders may help to prove that a design is correct with respect to a specific requirement.

\subsection{Dynamics}

In Section 2.1 the exchange of variables between systems defined their comparability. This does not mean, however, that all variables can change by exchange only, i.e. through an interaction such that equation (1) is satisfied. Yet there are variables for which this is the case. They are of immense importance since they satisfy a universal conservation law. This leads to the following classification of dynamical variables:

1. Variables that change their value by exchange only, that satisfy a universal conservation law. Processes changing such a variable need the participation of other systems.

2. Variables that may change in other ways but pure exchange. For those variables the processes can be divided into

(a) those during which the respective variable changes by exchange only, and

(b) all others.

In contrast to conservation one may talk of generation or production and of destruction or consumption. Processes that only change variables of the second kind do not necessarily depend on other systems: they may run 'automatically'.

Energy is the example of a variable of the first kind.

First Law. Energy can be exchanged only between dynamical systems; it cannot be generated or destroyed (conservation law).

Entropy is an example of a variable of the second kind. Its value can decrease by exchange only but may increase by generation as well.

Second Law. If during a process entropy decreases in one system $-\Delta S<0$ - then by the same process entropy must increase in another system by at least $|\Delta S|$.

One may say that entropy satisfies half of a conservation law. Processes which generate entropy cannot be reversed since the second law above excludes the destruction of entropy; they are irreversible. Processes conserving entropy, however, are reversible.

\subsection{Net Theory}

In this section four state quantities will be studied that have been identified for net systems in general, independent of any interpretation or application. The crucial question is whether they satisfy some conservation laws. And although the answer will be negative in all four cases, it seems worthwhile to understand the reasons. 
The four quantities are

- en-ability, $\mathscr{E}$, the characteristic function of net systems;

- decision content, $\mathscr{D}$, the result of choosing between possible modes of behaviour;

- information, $\mathscr{I}$, the quantitative measure for $\mathscr{D}$;

- enlogy, $\mathscr{L}$, closely intertwined with $\mathscr{I}$.

En-ability (23) has been discussed in Section 3.2; the other three quantities will be introduced a little further down in Section 8.2.2: enlogy (51), decision content (52) and information (53). None of these quantities are linear; they cannot be represented by forms. This must not surprise; if en-ability, for example, the characteristic function of net systems, were linear, net theory would just be part of the theory of linear systems.

\subsubsection{En-ability}

Obviously en-ability does not observe a conservation law in a strict sense. By an event occurrence any number of events may gain or lose en-ability. Yet the extensionality principle of net theory (see Section 3.2) that makes the en-ability the characteristic function of a net system seems to play a similar role that the conservation law of energy plays in the dynamics of physical systems. Hence the main consequences of the extensionality principle will be formulated as follows.

First Law. The enabling of an event is determined by its effect. An event gains its en-ability through the occurrence of some other event, and it loses its en-ability by either occurring itself or by the occurrence of some other, conflicting event.

\subsubsection{Enlogy and Information}

Roughly speaking, information is connected with choosing between modes of behaviour and enlogy with the resources needed for representing information. Using these terms we may talk informally about the example in Fig. 5 as follows:

- In state $\left\{s_{0}\right\}$ the system contains neither information nor enlogy.

- In the phase following the free choice $\left\{t_{1} \mid t_{2}\right\}$ two alternative states are possible: $\left\{s_{1}, s_{2}\right\}$ and $\left\{s_{3}, s_{4}\right\}$. Hence 1 bit of information has entered the system through the decision. (If there were a third outgoing transition leading into a third alternative state, $\log _{2} 3$ bit - one 'trit' - would enter the system; see also discussion of Fig. 7, below.

This information appears in our example in two copies that are to control concurrent subprocesses. However, the system does not contain two bits of - independent - information; the knowledge of one bit entails the knowledge of the other. We say that the system also contains 1 bit of enlogy and observe that the copying of information requires the same amount of enlogy.

- The two bits leave the system through the two concurrent merges (co-decisions) $\left\{t_{3} \mid t_{4}\right\}$ and $\left\{t_{5} \mid t_{6}\right\}$. State $\left\{s_{5}, s_{6}\right\}$ contains neither information nor enlogy.

The co-decisions are concurrent but not independent. In the reversed system they must be supplied with copies of the same bit of information; otherwise the reverse processes may deadlock.

- The four intermediate states $\left\{s_{1}, s_{6}\right\},\left\{s_{2}, s_{5}\right\},\left\{s_{3}, s_{5}\right\}$ and $\left\{s_{4}, s_{6}\right\}$ still hold one bit of information but no enlogy anymore.

Hence it is impossible to say by which of the two co-decisions the enlogy and the information leaves.

It seems very difficult to define enlogy $\mathscr{L}$ for net systems in general. Hence the following considerations are restricted to a special class which will be called synchronisation schemes. They combine synchronisation graphs (marked graphs) [CHE71, GeL73] and PrT nets [GeL81]. Their purpose is that choices will depend only on the values of tokens rather than on the distribution of tokens as well.

Synchronisation schemes are defined by the following restrictions.

1. Every place

- either has exactly one incoming and one outgoing arc

- or its input and output transitions are the same - it forms a set of loops. 

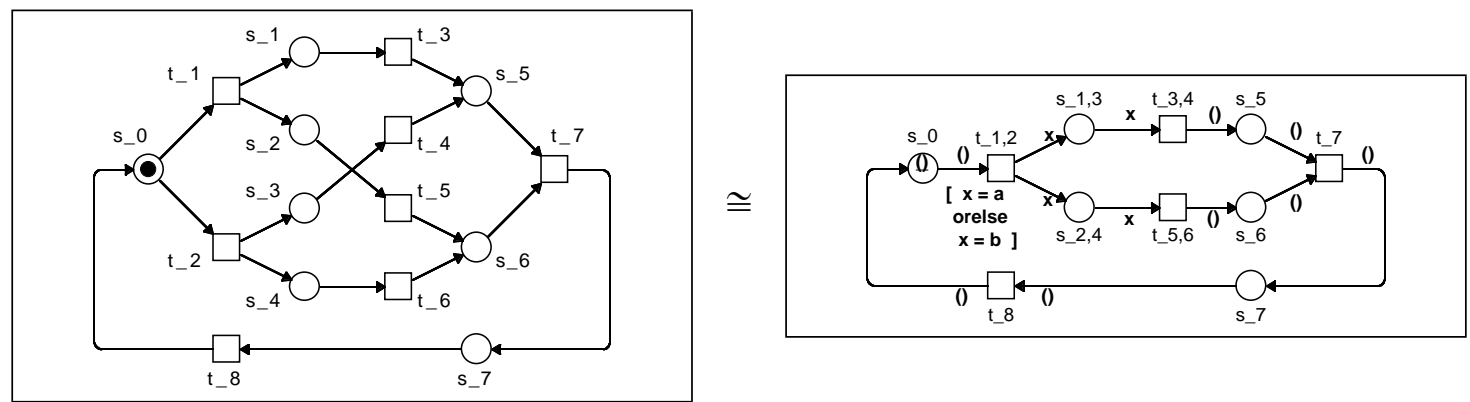

Fig. 5. Net system with a free choice between modes.
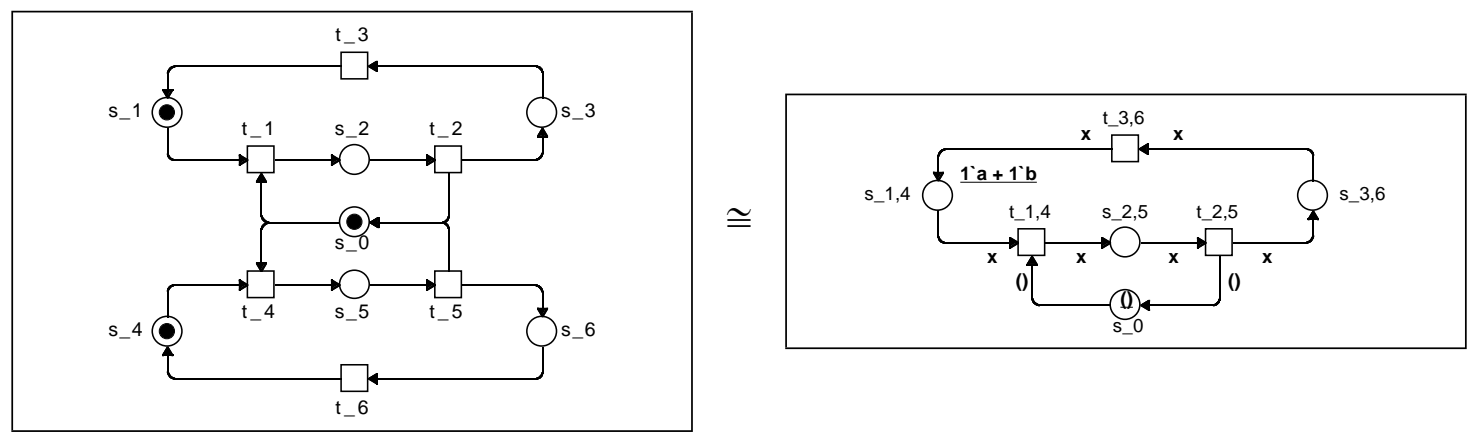

Fig. 6. Net system with a race between two modes.

2. The subnet without shared places is strongly connected.

3. All colour sets are finite.

4. All arc expressions denote a single token; there no are multiple arcs.

5. The behaviour is cyclic: the reachability graph is strongly connected.

The 'grey' markings of a synchronisation scheme where the tokens are not distinguished by their values but counted only are called its phases. The grey markings of the shared places do not change. The subnet without shared places is called its flow graph; under the phases it forms a live and bounded synchronisation graph.

Two known subclasses of synchronisation schemes are information flow graphs [Pet67] and bipolar synchronisation schemes [GeT84]. Both classes have live and safe (1-bounded) underlying synchronisation graphs; in both classes all places have the same binary colour set. Another subclass consists of the marked-graph-like $\operatorname{PrT}$ nets [LaP85].

The two net systems on the left of Figs 5 and 6 can be transformed into equivalent synchronisation systems (on the right) through a sequence of equivalence transformations of PrT nets [Gen90]. In both cases the colour sets consist of at most two elements. The free-choice example is 1-bounded; the race example is 2-bounded. The production scheme (Fig. 3) is a non-example in several respects. Two places are shared; two arc expressions contain Boolean coefficients; and without the border transitions $t_{0}$ and $t_{6}$, it is not cyclic.

Next to the phases the concept of a mode of behaviour [Fur76] is needed. Its definition does not necessarily depend on synchronisation schemes but modes are particularly simple there.

Let $Z$ be an elementary cyclic process of a synchronisation scheme $\Sigma$ and $z$ the corresponding T-vector of combinations of bindings; then $C \cdot z=0$ for the incidence matrix $C$. The T-vector $z$ is called a mode of $\Sigma$; the set of all modes is denoted as $\mathscr{M}(\Sigma)$. Every elementary solution of $C \cdot z=0$ with non-negative integer coefficients that can be executed is a mode. In an execution of a mode of a synchronisation scheme, all transitions occur the same number of times.

For a particular marking $M$, the set of modes that can be executed starting at $M$ reflects the degree of non-determinism of $M$; it is denoted by $\mathscr{M}(M)$. The examples in Figs 5 and 6 both have two modes which are folded on top of each other to get the synchronisation scheme. Figure 7 has three modes that have to be unified first before the synchronisation scheme can be formed. 

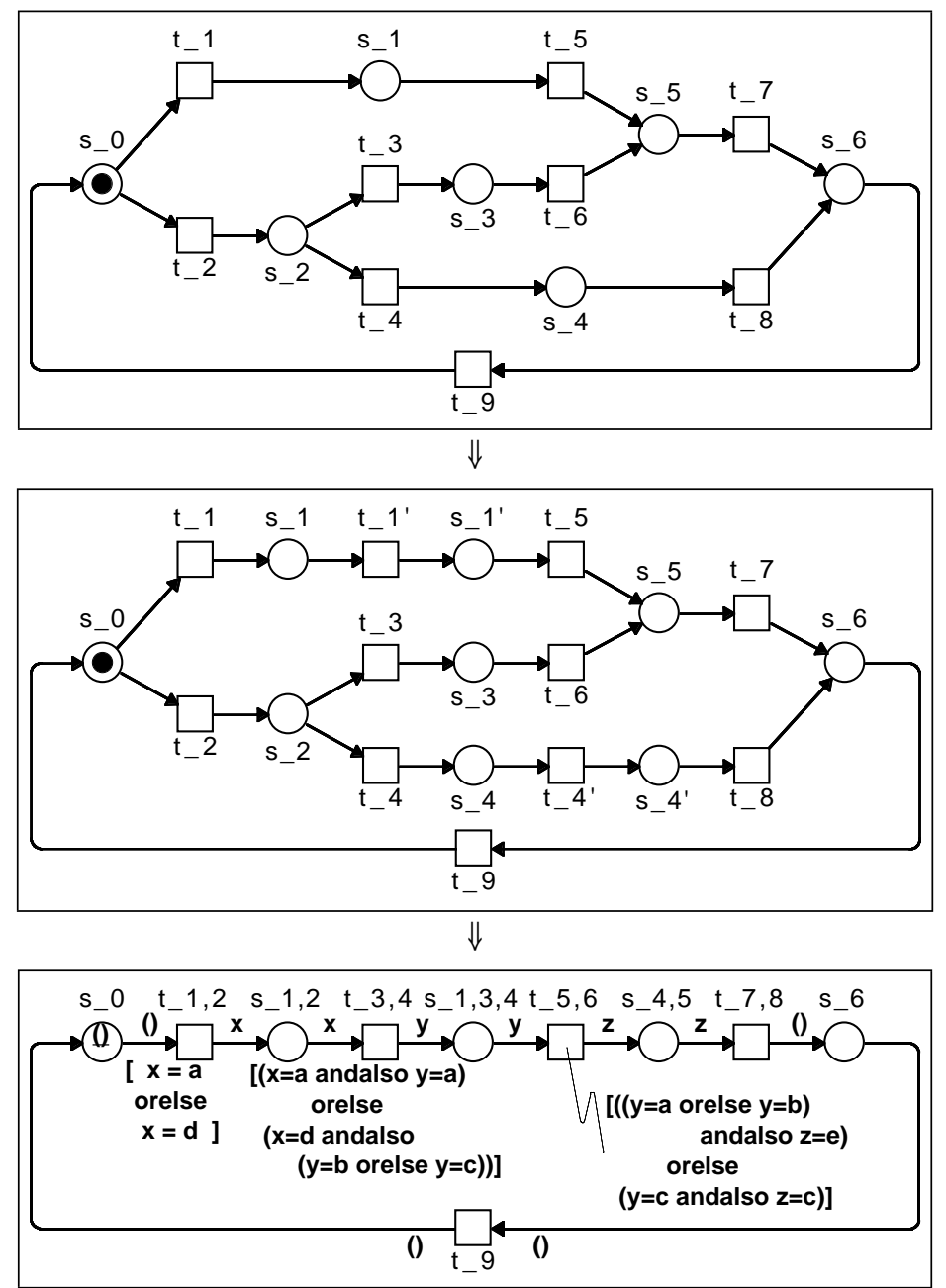

Fig. 7. Folding of an unnested double if-then-else.
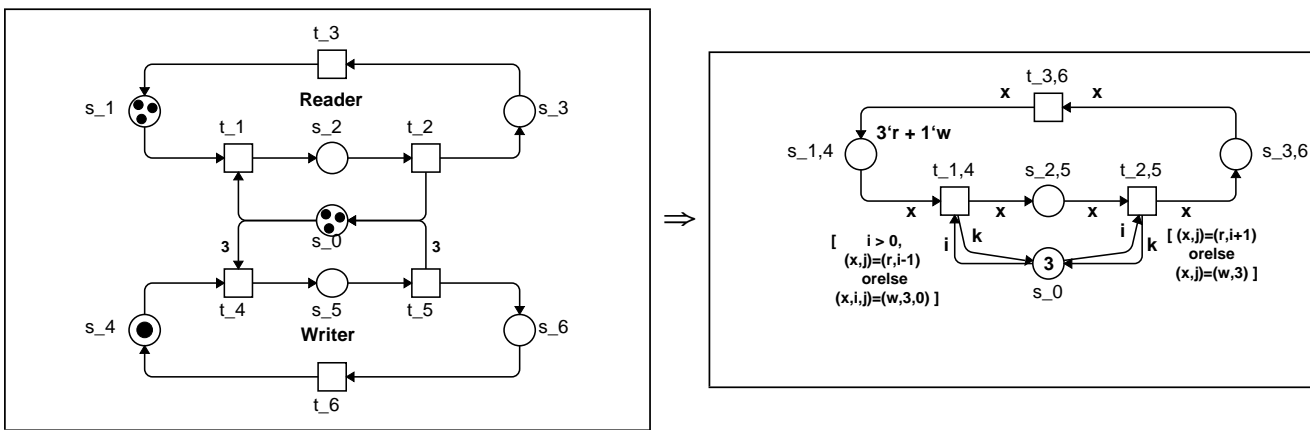

Fig. 8. Transformation of a simple reader-writer scheme.

The three examples above can be transformed into mere flow graphs, without shared places. This is not true for the generalisation of the - symmetric - race in Fig. 6 to the asymmetric reader-writer example (Fig. 8). Here the write mode has an arc multiplicity greater than one. The transformation into a synchronization scheme requires a proper resource allocation scheme [Sha79]. 

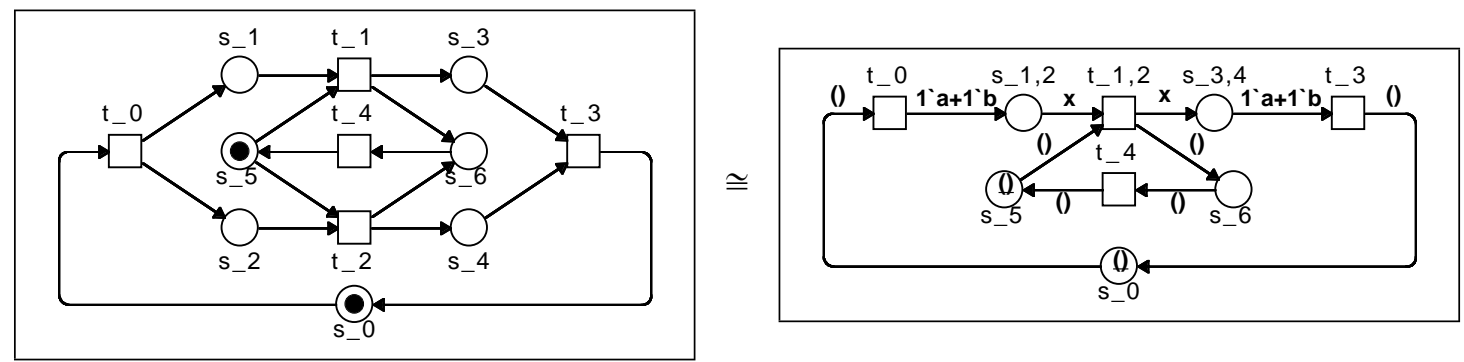

\begin{tabular}{c|ccccc}
$c$ & $\left\{s_{0}, \ldots\right\}$ & $\left\{s_{1}, s_{2}, \ldots\right\}$ & $\left\{s_{1}, s_{4}, \ldots\right\}$ & $\left\{s_{2}, s_{3}, \ldots\right\}$ & $\left\{s_{3}, s_{4}, \ldots\right\}$ \\
\hline $\mathscr{L}$ & 0 & 0 & 1 & 1 & 0 \\
$\mathscr{I}$ & 0 & 0 & 1 & 1 & 0
\end{tabular}

Elementary net system (left)

\begin{tabular}{c|ccccc}
$M$ & $\left\{s_{0}, \ldots\right\}$ & $\left\{s_{1,2}=a+b, \ldots\right\}$ & $\left\{s_{1,2}=a, s_{3,4}=b, \ldots\right\}$ & $\left\{s_{1,2}=b, s_{3,4}=a, \ldots\right\}$ & $\left\{s_{3,4}=a+b, \ldots\right\}$ \\
\hline $\mathscr{L}$ & 0 & 1 & 1 & 1 & 1 \\
$\mathscr{I}$ & 0 & 0 & 1 & 1 & 0
\end{tabular}

Synchronisation system (right)

Fig. 9. Representation-dependent enlogy (variant of the race example).

\section{Enlogy}

Let $\left(\Sigma, M^{0}\right)$ be a synchronisation scheme with a given initial marking. Let $\tau$ be one of its phases (grey markings), $\tau=\left\{g_{1}, \ldots, g_{n}\right\}$. For each of the grey tokens $g_{i}$ the data type $D_{i}$ of the corresponding place determines the range of values that $g_{i}$ may assume. Hence the set of colorations of $\tau$ that are possible in principle is the Cartesian product $\prod_{i=1}^{n} D_{i}$. Let $\mu(\tau)$ denote the colorations of $\tau$ that actually belong to the marking class $\left[M^{0}\right]$. Usually it is a much smaller subset.

The ratio between the number of markings feasible at phase $\tau$ and the number of those markings that are actually possible (reachable) at $\tau$ is a measure of the order imposed by the system. Its logarithm to base 2 is called the enlogy of (all states at) phase $\tau$ and denoted by $\mathscr{L}$; the unit is bit. ${ }^{11}$

$$
\mathscr{L}(M)=\log _{2} \frac{\prod_{i=1}^{n}\left|D_{i}\right|}{|\mu(\tau)|} \quad(M \in \mu(\tau))
$$

In the free-choice example in Fig. 5, the phase $\left\{s_{1,3}, s_{2,4}\right\}$ following the decision allows four different colorations but only two are system states. The enlogy is $\log _{2} \frac{4}{2}=1$ bit as told in our narration above.

Assume that at a phase $\tau$, the data type $D$ of a grey token has more elements than actually appear in any of the reachable markings of $\tau$. (The reason could be that the net is to represent a whole family of systems by virtue of different initial markings.) Then the additional - redundant - enlogy reflects the extra cost of implementing $D$. Enlogy is a representation dependent quantity.

This is not a matter of economy only. Look at a variant of the race example that is shown in Fig. 9. In the elementary net system, the event $t_{0}$ does not add enlogy while in the equivalent synchronisation scheme, it adds 1 bit.

En-ability and information, however, are preserved by the equivalence transformations of PrT nets which are based on the isomorphism of reachability graphs [Gen90].

\footnotetext{
$\overline{11}$ Enlogy and its relative, enlogic structure, were coined by C. A. Petri [Pet67, Pet76].
} 


\section{Decision Content and Combinatorial Information}

The set of all modes of a system, $\mathscr{M}(\Sigma)$, indicates the amount of behavioural freedom in $\Sigma$. The more modes are possible at a particular state $M$, i.e. the larger the set $\mathscr{M}(M)$ is, the more information is needed in order to predict what may happen next. Conversely, the more modes are excluded at $M$, i.e. the larger the set $\mathscr{M}(\Sigma)-\mathscr{M}(M)$ is, the larger is the decision content of $M$. The quantity

$$
\mathscr{D}(M)=\mathscr{M}(\Sigma)-\mathscr{M}(M)
$$

is called the - combinatorial - decision content of state $M$. The quantity

$$
\mathscr{I}(M)=\log _{2} \frac{|\mathscr{M}(\Sigma)|}{|\mathscr{M}(M)|}
$$

is called the - combinatorial - information of state $M$; its unit is the bit. ${ }^{12}$

The examples in Figs 5 and 6 both have two modes: $|\mathscr{M}(\Sigma)|=2$. At the initial states $\left\{s_{0}\right\}$ and $\left\{s_{0}, s_{1,4}\right\}$ none of the modes is excluded; the information is $\mathscr{I}\left(M^{0}\right)=\log _{2} \frac{2}{2}=0$ bit. The decisions $t_{1,2}$ and $t_{1,4}$ respectively exclude one mode: $\left|\mathscr{M}\left(M^{1}\right)\right|=1$. One bit of information enters the system, $\mathscr{I}\left(M^{1}\right)=\log _{2} \frac{2}{1}=1$ bit.

The example in Fig. 7 has three modes. At the initial state $\left\{s_{0}\right\}$ no mode is excluded; the information is 0 bit. Depending on the outcome of decision $t_{1,2}$ either one or two modes are excluded. The information added is $\log _{2} \frac{3}{1}$ bit for $x=a$ and $\log _{2} \frac{3}{2}$ bit for $x=d$. Decision $t_{3,4}$ adds either no information $(x=y=a)$ or exactly one bit $(x=d \wedge(y=b \vee y=c))$. In both cases, the information becomes $\left(\log _{2} 3=\log _{2} \frac{3}{2}+\log _{2} \frac{2}{1}\right)$ bit. In a mirrored way the information leaves the system.

The production scheme in Fig. 3 has a mode for every feasible batch. ${ }^{13}$ The actual information flow depends on a number of parameters. At $t_{1}$ the batches may race for the available resources. For the two batches in the given initial marking the available devices just suffice. There is no conflict; they can be produced concurrently. If more orders are accepted races may occur and information is needed to resolve them.

As a last example assume a synchronisation scheme where at all transitions the binding of the individual variables at the output arcs is a function of the binding of the variables at the input arcs. Such a system has only one mode. The information is constant zero; the system is closed with respect to information flow. A special case are the live and bounded marked graphs where the data type of all places is unit $=\{()\}$. If we had tried - because of its role in 'information processing' - to use information as a characteristic function for net systems, any two net systems closed under information flow with the same set of typed places would be considered the same.

Whenever a conflict is resolved in a net system $\Sigma$, the information contained in $\Sigma$ increases. And if $\Sigma$ is cyclic, the information will decrease eventually. Only conflict-free systems are closed under information flow. If by a closer look into the environment of $\Sigma$ the outcome of a decision (conflict resolution) can be predicted, then the environment contains the information. It enters $\Sigma$ either by exchange $(f l u x)$ or by copying (influence) [Pet67]. If, on the other hand, the source of the information is inaccessible, if even the most precise reconstruction of the situation before the decision cannot guarantee the same outcome, then there is a random decision and information is generated. This is in contradiction to the principle of local determinacy [Pet77] stating that if a conflict is resolved in a net system, the information comes from an environment.

Examples. The numbers of the national lottery; the decision of a close race, like an asynchronous input or a shared resource allocation, by an arbiter; analogue-to-digital conversion ('fuzzification') close to the border between two intervals.

In general, conflict resolution in the presence of confusion [Smi96] requires the generation of information. And since the holding of information needs resources and resources are limited, information that is generated must be destroyed eventually. Information does not even satisfy half of a conservation law like entropy as we might have hoped.

Second Law. The decision of a race, i.e. of a conflict in a situation of confusion, requires the generating of information. The limitation of resources requires the destroying of information (recycling).

\footnotetext{
12 Combinatorial decision content and information were first coined by Holt and Commoner in [HoC70]

13 In practice, an upper bound for the size of batches keeps the number of modes finite.
} 


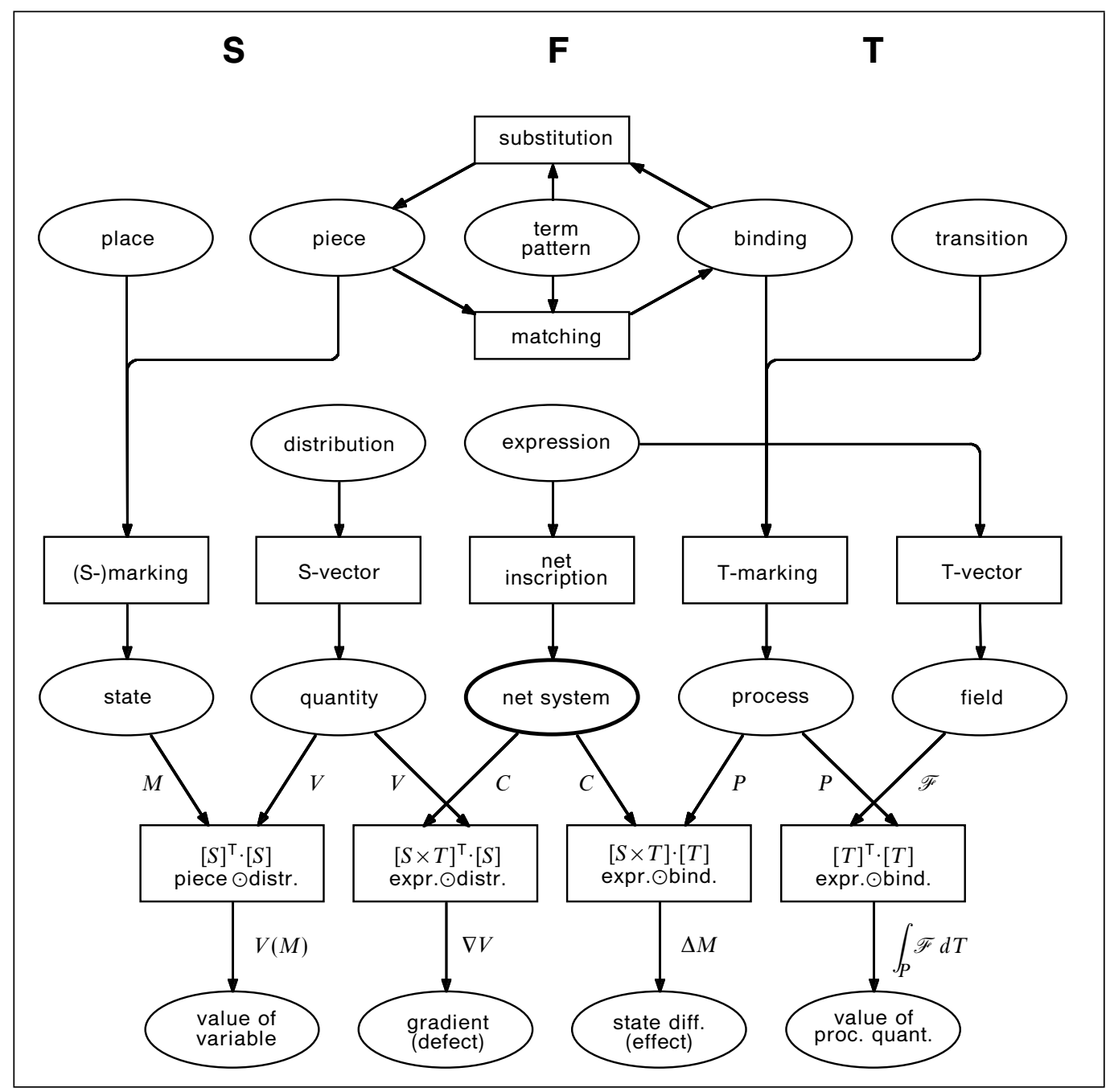

Fig. 10. The dynamics of net systems - survey.

\section{Information Work}

The information work done during a process $M_{1} \stackrel{P}{\longrightarrow} M_{2}$ is

$$
W_{\mathscr{I}}=\Delta \mathscr{I}=\mathscr{I}\left(M_{2}\right)-\mathscr{I}\left(M_{1}\right)
$$

The free choice example in Fig. 5 demonstrates that by the same event, depending on the current state, either information or enlogy may leave the system. This is due to the fact that several copies of the same bit of information may exist concurrently.

The consequence is that we cannot, in general, tell which information work is done by which event. Information work has no forms, in general; the work done by a process, for example the cost of computation [War68], cannot be calculated as the line integral over a field (of 'information forces'). When looking at earlier work on modes, decision content and information in net systems [Fur76, Bes82], it seems that the intricate interplay of information and enlogy could not be understood without higher-level nets and their equivalence transformations. And the thermodynamical approach taken in [Joh88] may only work if combinatorial information is replaced by statistical information as in information theory. 
Table 7. General dynamics and net theory: similarities and differences.

\begin{tabular}{|c|c|c|}
\hline & Physical systems & Net systems \\
\hline Modeling & physical, continuous & informatical, discrete \\
\hline Coordinates & independent state variables & places \\
\hline Values & real number·unit & $\sum($ number $\cdot$ piece $)$ \\
\hline State & vector of coordinate values & $\begin{array}{l}\text { set of coexisting conditions } \\
\text { (case, marking) }\end{array}$ \\
\hline Change & $\begin{array}{l}\text { infinitesimal change } d x \\
\text { (of a coordinate } x \text { ) }\end{array}$ & $\begin{array}{l}\text { elementary change (event) } \Delta t \\
\text { (at a transition } t \text { ) }\end{array}$ \\
\hline Process & $\begin{array}{l}\text { continuous sequence of states } \\
\text { (parameter time) }\end{array}$ & $\begin{array}{l}\text { set of event occurrences with } \\
\text { partial order earlier }\end{array}$ \\
\hline Time & parameter of process & $\begin{array}{l}\text { events: time points } \\
\text { conditions: non-zero durations }\end{array}$ \\
\hline $\begin{array}{l}\text { Coordinate } \\
\text { transf'ns }\end{array}$ & $\begin{array}{l}\text { equivalence of different } \\
\text { coordinate systems }\end{array}$ & $\begin{array}{l}\text { equivalence transformations } \\
\text { of PrT nets }\end{array}$ \\
\hline $\begin{array}{l}\text { Characteristic } \\
\text { function }\end{array}$ & $\begin{array}{l}Y=Y\left(x_{1}, \ldots, x_{n}\right) \\
\text { (energy preferred) }\end{array}$ & $\begin{array}{l}\mathscr{E}(c)=\left\{e \mid \bullet-e^{\bullet} \subseteq c \wedge e^{\bullet}-\bullet \subseteq \bar{c}\right\} \\
\text { en-ability (imperative) }\end{array}$ \\
\hline Forms & $\begin{array}{l}\frac{\partial Y\left(x_{1}, \ldots, x_{n}\right)}{\partial x_{i}} d x_{i} \\
\quad(\text { variable } Y)\end{array}$ & $\begin{array}{c}\frac{\partial V\left(s_{1}, \ldots, s_{n}\right)}{\partial s_{i}} \Delta s_{i} \\
(\text { linear variable } V) \\
\frac{\partial W\left(t_{1}, \ldots, t_{m}\right)}{\partial t_{j}} \Delta t_{j} \\
\text { (linear process quantity } W \text { ) }\end{array}$ \\
\hline Composition & $\begin{array}{l}\text { addition of comparable } \\
\text { characteristic functions }\end{array}$ & addition of disjoint nets \\
\hline Coupling & $\begin{array}{l}\text { exchange of coordinates, } \\
x^{a}+x^{b}=\text { const., } x^{a} \text { free }\end{array}$ & $\begin{array}{l}\text { fusion of places } \\
\text { fusion of transitions }\end{array}$ \\
\hline Flow & exchange & $\begin{array}{l}\text { flux (exchange) and } \\
\text { influence (copying) }\end{array}$ \\
\hline $\begin{array}{l}\text { Laws of } \\
\text { dynamics }\end{array}$ & $\begin{array}{l}1^{\text {st }} \text { law: energy } \\
2^{\text {nd }} \text { law: entropy }\end{array}$ & $\begin{array}{l}\text { extensionality principle (en-ability) } \\
\text { generation of information } \\
\text { in situations of confusion }\end{array}$ \\
\hline
\end{tabular}

\section{Conclusion}

This paper presented some known material of higher-level Petri nets from a particular perspective, namely in analogy to the dynamics of physical systems. The aim was to widen the view on the role of the incidence matrix in the analysis of net systems. Rather than focusing on constant state quantities (S-invariants) and cyclic actions (T-invariants), i.e. on solutions of the homogeneous equation systems based on the incidence matrix, we propose the study of dynamical quantities of net systems in general.

Figure 10 shows the main ingredients of the dynamical analysis of net systems. It exhibits the typical $S-T$ symmetry resulting from the fact that net theory treats the notions state and change on an equal footing. This symmetry is reflected not only by the linear-algebraic duality between the incidence matrix $C$ and its transpose, but it also appears closely related to the two complementary forms in which data appear as structured sets of values. As pieces, data are stored and transmitted - 'displaced' over distances in time and space; as bindings, data are processed and combined with other data. As pieces they belong to the states, as bindings they belong to the processes.

Table 7 summarises our comparison of the general dynamics of physical systems to a potential net dynamics that was outlined in this paper. It shows some promising similarities as well as severe differences.

The decomposition into forms, in analogy to the forms of energy, is limited to linear quantities. The main dynamical quantities of net systems, however, en-ability, information and enlogy, are non-linear. 
There is no equivalent to the first law of dynamics (conservation of energy) nor to the second law (semi-conservation law for entropy). However, the situations in which information is created can be localised. They are situations of confusion [Smi96] where the decision of races requires special elements with meta-stable states (arbiters).

Information work in general cannot be expressed in terms of forms associated with the transitions, not even for the special class of synchronisation systems, as was shown by the analysis of the intricate interplay of information and enlogy in Section 8.2. Calculation of the information content of states and of the information work done in the course of processes requires to determine the set of all possible modes of behaviour through the analysis of the reachability graph - quite an unpleasant fact.

In spite of these difficulties the analysis of net systems in terms of dynamical quantities, supported by an adequate computer-algebra tool, seems a worthwhile endeavor.

\section{Acknowledgements}

The author is greatly indebted to Dan Simpson and to the referees who - with one exception - encouraged and helped him to improve the quality of this paper.

\section{References}

[Bes82] Best, E.: Information flow in nets. In C. Girault and W. Reisig, editors. Application and Theory of Petri Nets. InformatikFachberichte 52, pages 82-87. Springer, Berlin, 1982.

[ChH94] Christensen, S. and Hansen, N. D.: Coloured Petri nets extended with channels for synchronous communication. In R. Valette, editor. Application and Theory of Petri Nets. Lecture Notes in Computer Science, Vol. 815, pages 159-178. Springer, Berlin, 1994

[CHE71] Commoner, F., Holt, A. W., Even, S. and Pnueli, A.: Marked directed graphs. Journal of Computer and System Science 5: 511-523, 1971.

[CPN96] Design/CPN Reference Manual, Version 3.00. DAIMI, Aarhus University, 1996, www.daimi.au.dk/designCPN/

[Fa166] Falk, G.: Theoretische Physik auf der Grundlage einer allgemeinen Dynamik. Band I: Punktmechanik. Band II: Allgemeine Dynamik, Thermodynamik. Springer, Berlin, 1966/1968

[Fur76] Furtek, F. C.: The logic of systems. Technical Report 170. MIT-LCS, Cambridge, MA, June 1976.

[Gen90] Genrich, H. J.: Equivalence transformations of PrT-nets. Advances in Petri Nets 179-208, 1990.

[GeH95] Genrich, H. J. and Hanisch, H.-M.: Modelling and analysis of recipes. In Workshop on Analysis and Design of Event-driven Operations in Process Systems, Imperial College, April 1995.

[GeL73] Genrich, H. J. and Lautenbach, K.: Synchronisationsgraphen. Acta Informatica 2: 143-161, 1973.

[GeL81] Genrich, H. J. and Lautenbach, K.: System modeling with high-level Petri nets. Theoretical Computer Science 13: 109-136, 1981.

[GeT84] Genrich, H. J. and Thiagarajan, P. S.: A theory of bipolar synchronization schemes. Theoretical Computer Science 30: 241-318, 1984.

[HoC70] Holt, A. W. and Commoner, F.: Events and Conditions. Information System Theory Project. Applied Data Research Inc., Princeton, NJ, 1970.

[Jen81] Jensen, K.: Coloured Petri nets and the invariant method. Theoretical Computer Science 14: 317-336, 1981.

[Jen92] Jensen, K.: Coloured Petri Nets. Volume 1: Basic Concepts. Volume 2: Analysis Methods. Volume 3: Practical Use. Springer, Berlin, 1992|1995|1997.

[Joh88] Johnson, R. R.: Elements of a theory for computer performance. Technical Report. University of Utah, Salt Lake City, 1988.

[LaP85] Lautenbach, K. and Pagnoni, A.: Liveness and duality in marked-graph-like predicate/transition nets. Advances in Petri Nets 331-352, 1980.

[Pau91] Paulson, L. C.: ML for the working programmer. Cambridge University Press, Cambridge, 1991.

[Pet62] Petri, C. A.: Kommunikation mit Automaten. Schriften des IIM Nr. 2. Rheinisch-Westfälisches Institut für Instrumentelle Mathematik an der Universität Bonn, Bonn, 1962.

[Pet67] Petri, C. A.: Grundsätzliches zur Beschreibung diskreter Prozesse. In W. Händler, E. Peschl and H. Unger, editors. 3. Colloquium über Automatentheorie, pages 121-140. Birkhäuser, Basel, 1967.

[Pet76] Petri, C. A.: Interpretations of net theory. Technical Report ISF 75-07. GMD, St Augustin, 1976.

[Pet77] Petri, C. A.: General Net Theory. In B. Shaw, editor. Computing System Design, pages 131-169. University of Newcastle, 1977 .

[Pet82] Petri, C. A.: State-transition structures in physics and in computation. International Journal of Theoretical Physics, 21: 979-992, 1982.

[Sha79] Shapiro, R. M.: Towards a design methodology for information systems. In C. A. Petri, editor. Ansätze zur Organisationstheorie rechnergestützter Informationssysteme. Berichte der GMD Nr. 111, pages 107-118. Oldenbourg, München, 1979 .

[Smi96] Smith, E.: On the border of causality: contact and confusion. Theoretical Computer Science, 153: 245-270, 1996.

[War68] Warshall, S.: The cost of computation. In A. W. Holt et al., editors. Information System Theory Project: Final Report. RADC-TR-68-305. Applied Data Research, Inc., Princeton, NJ, September 1968. 


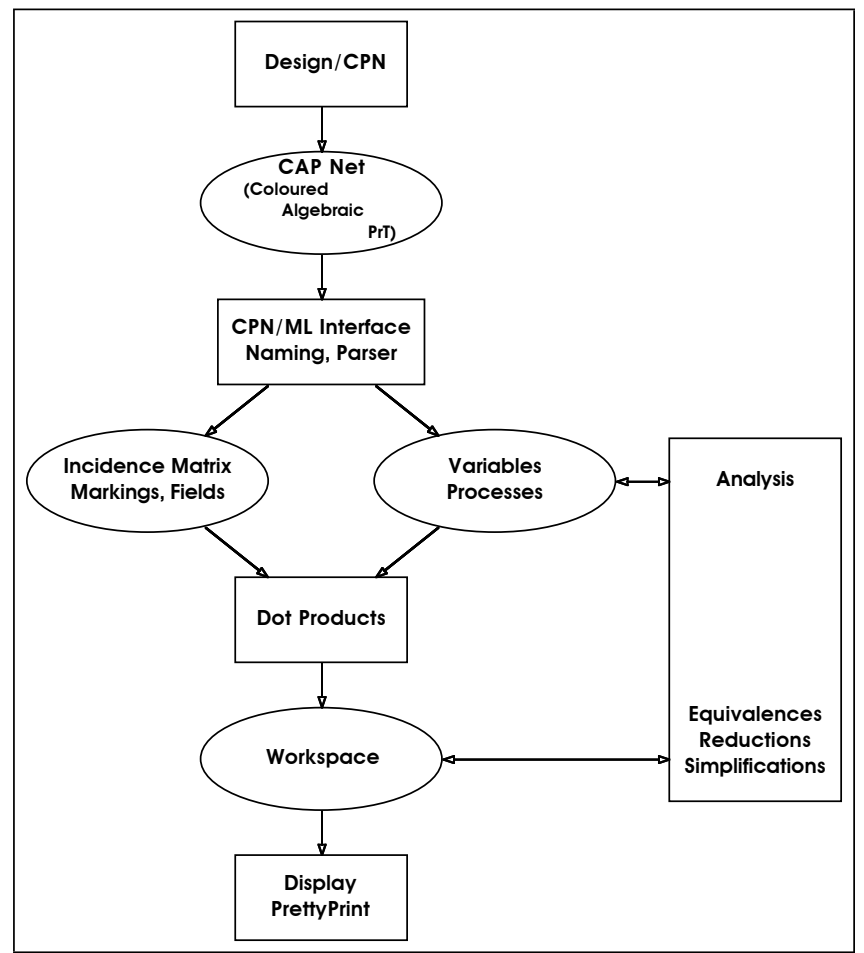

Fig. 11. Structure of the analysis package.

\section{A. Appendix: Symbolic Calculations}

To complement the theoretical parts of this paper, this Appendix briefly describes the experimental software package that supported symbolic calculations based on the incidence matrix like those in Sections 6.2 and 7.2. The package is still quite experimental. Its original purpose was to conduct some experiments concerning the purely symbolic or computer-algebraic analysis of higher-level net systems - the kind K. Lautenbach and the author once envisioned after their discovery of predicate transition nets but failed to realise by taking an unsuitable approach (solving equations in a ring of polynomials, cf. [GeL81]).

When the package became useful enough to do some non-trivial computations, it had a great impact on the dynamical view of net systems presented in this paper and thence, in return, developed a broader purpose and had to undergo many revisions.

\section{A.1. An Experimental Analysis Package}

The tool is an addition to Design/CPN[CPN96] and has been written in Standard ML [Pau91]. Its overall structure is depicted in Fig. 11.

1. By means of the Design/CPN's $M L$ interface to the graphical model and its database, the executable $C P N$ model is translated into an equivalent incidence matrix representation. The net inscriptions (character strings) are transformed into an internal representation (kind of syntax tree) facilitating symbolic manipulation.

2. Dot product and matrix product are implemented based on the product between expression and transformation - the application of (combinations of) transformations on (combinations of) expressions.

3. A large number of reduction rules for combinations of expressions (equivalences, simplifications) are built in; they can be switched on and off interactively. 
4. The (intermediate) results of a calculation are kept in a workspace IT. There are several formats for them to visualise.

5. Form sheets derived from the model facilitate the definition of markings and processes, of variables and fields, of functions and parameters.

6. The built in general reduction rules can be augmented by user-defined rules and declarations written in $M L$.

\section{A.2. The Incidence Matrix}

The incidence matrix is the non-hierarchical equivalent of the executable version of the CPN model. It is created while Design/CPN is in its simulator state. Our example is the production scheme, Fig. 3 with border transitions omitted and time being ignored.

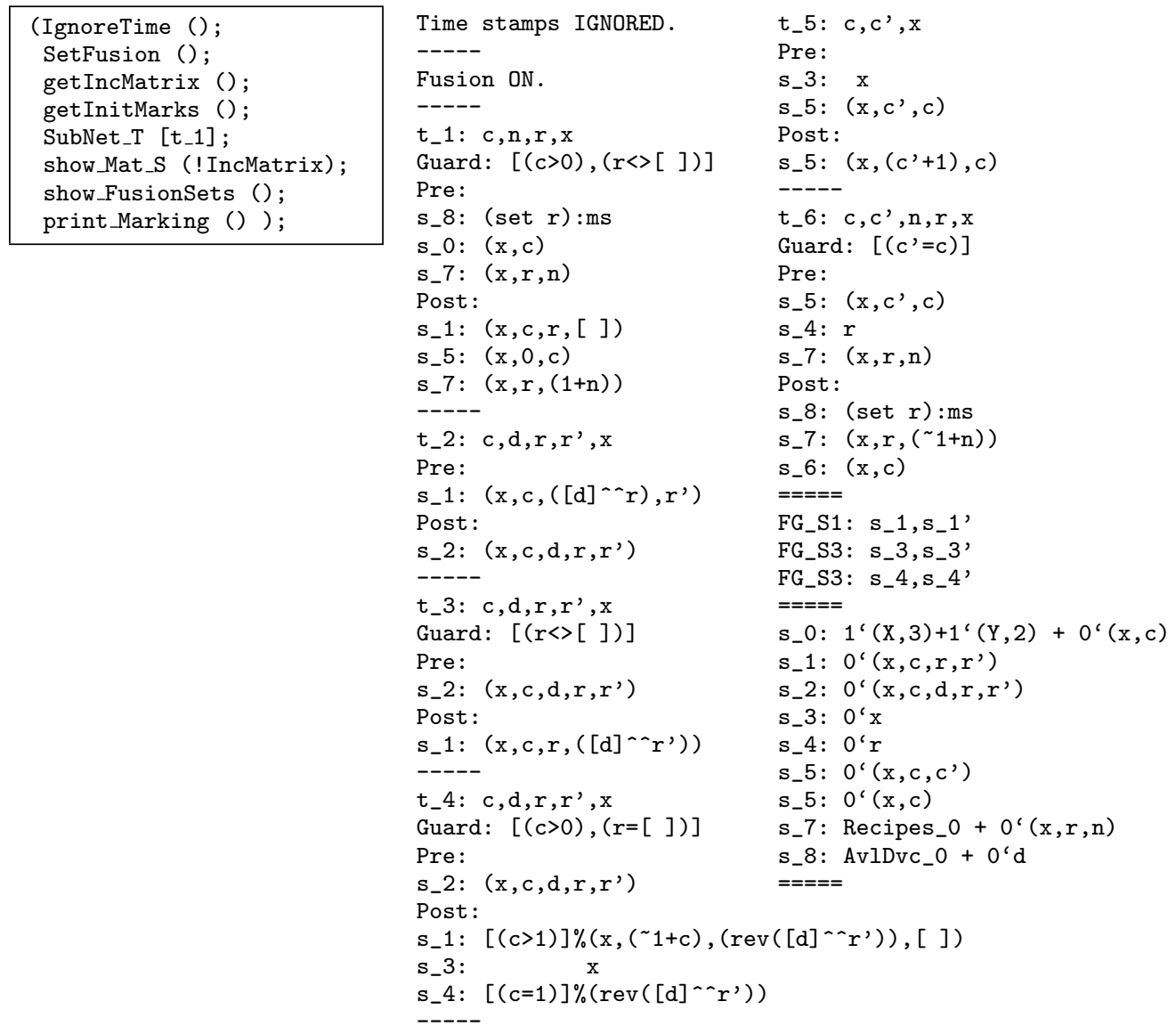

The representation is transition based (column-wise). In addition to the arc expressions in the pre and post, the guard and the free individual variables (the binding type) of each transition are listed. The naming conventions for multiple instances of places and transitions in a hierarchical model are those of the occurrence graph analyser of Design/CPN. The elements of place fusion sets are listed separately.

When this read-only master matrix has been created, the simulator state can be left. The actual analysis operates on two temporary working copies - primal and transposed form - derived from the master matrix. Submatrices based on subsets of places and transitions can be formed, and place fusion can be switched on and off. 


\section{A.3. Processes}

The effect of a process $M_{1} \stackrel{P}{\longrightarrow} M_{2}$ is the marking difference $\Delta M=M_{2}-M_{1}$. It is calculated as the matrix product between the incidence matrix $C$ and the action (one-column matrix) performed by $P$ (see equation $(15))$.

Let $P$ be the process of executing the order 'three times product $X$ ' represented by the token $(X, 3)$ in the initial marking of place $s_{0}$. Combining the events (bindings) at each transition yields the action action_ $x_{-} 3$. The product of $C$ with action_ $x_{-} 3$ is first shown in non-reduced form. Applying the built-in reduction rules for Boolean coefficients, numbers and lists yields the net effect of action_x_3 as expected.

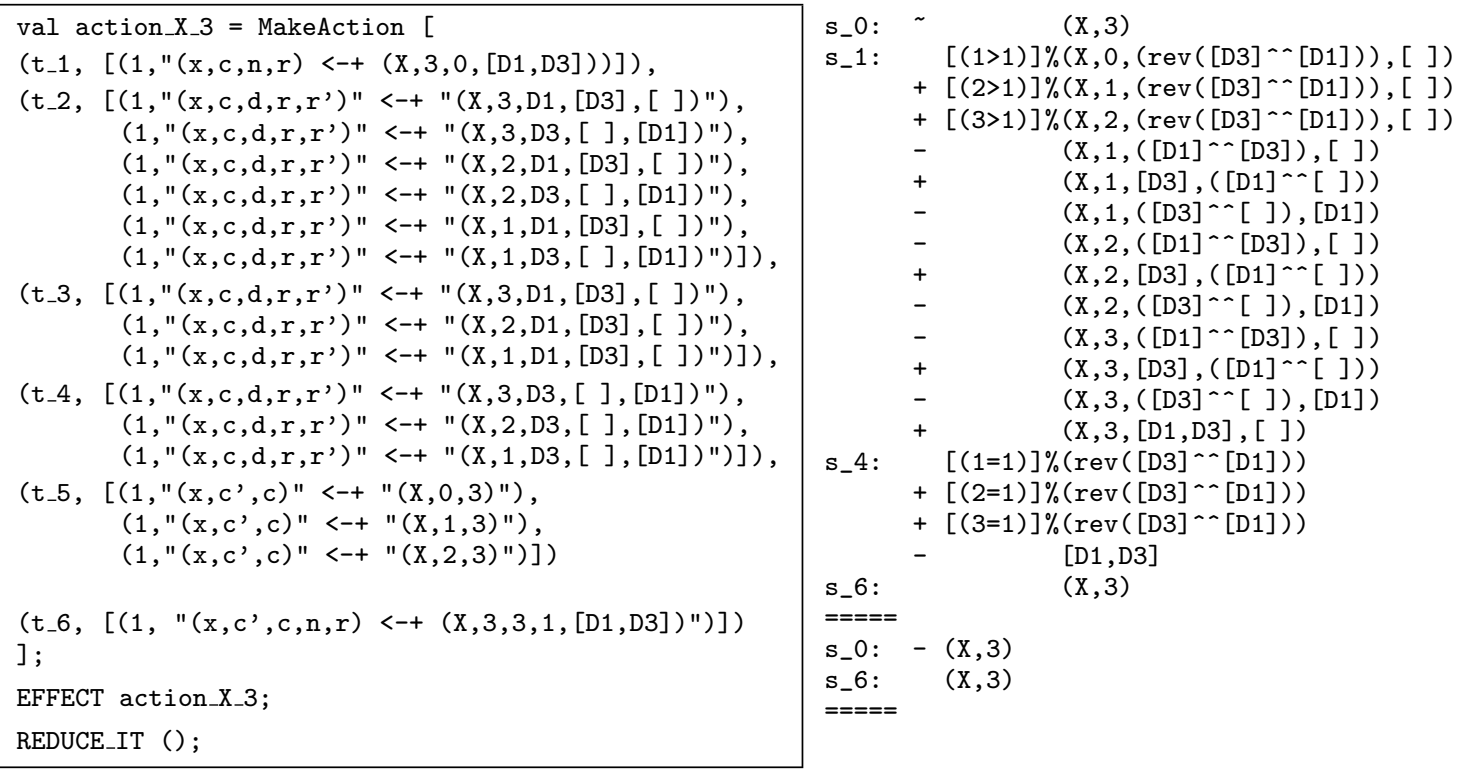

\section{A.4. State Quantities and their Gradients}

Next we demonstrate how to fill the gaps that were left in Section 6.2 when calculating the gradient of the quantity recipe.

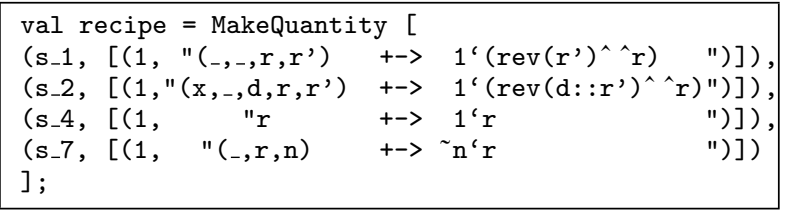

The gradient of recipe, the vector of its partial derivatives with respect to the transitions, is calculated as the matrix product of the transposed incidence matrix, $C^{\top}$, and the vector recipe viewed as a one-column matrix. The raw, non-reduced result is simplified considerably by means of the reduction rules for list expressions. 


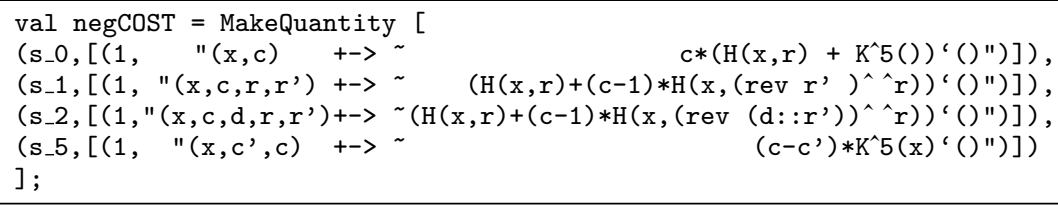

The next step consists of three substeps:

1. The gradient of $n \operatorname{eg} \operatorname{COS} T=-\operatorname{COS} T$ is calculated. The lengthy, non-reduced intermediate result we know already from section 7.2; it shrinks considerably when the built-in reduction rules are applied.

2. The partial derivative at $t_{4}$ is reduced by exploiting its guard. First the identity $[c>1]=[$ true $]-[c=1]$ that follows from $c>0$ is applied once again; then the equation $r=\left[\right.$ ] is applied at $t_{4}$ as well as $c^{\prime}=c$ at $t_{6}$.

3. Only now the definition of the - recursive - auxiliary function $H$ is provided and evaluated.

4. The final result is exactly the field cost as defined above.

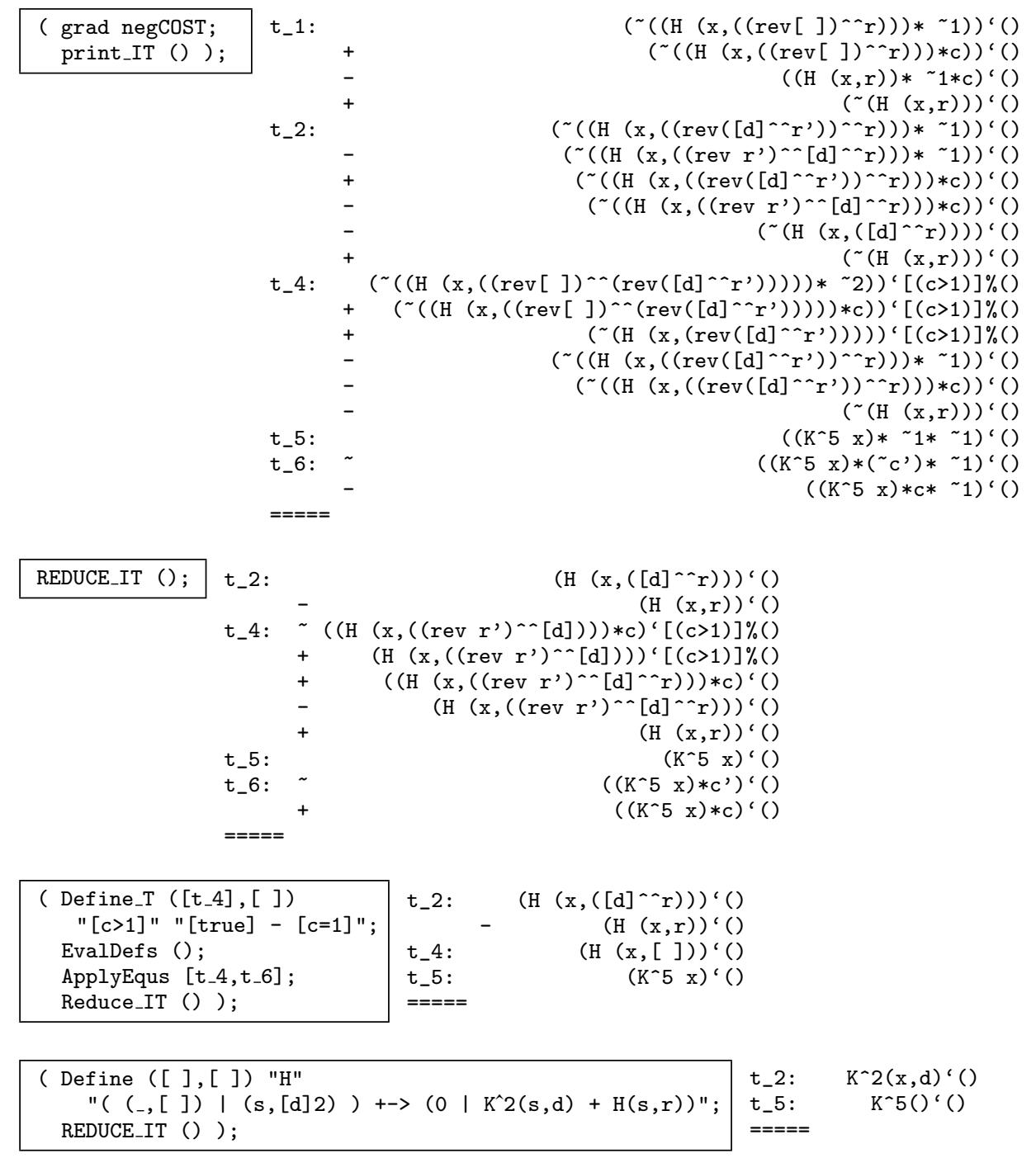

Received March 2000

Accepted in revised form March 2002 by D Simpson 